ARTICLE

https://doi.org/10.1038/s41467-019-12032-9

\title{
OPEN
}

\section{Recurrent GNAQ mutation encoding T96S in natural killer/T cell lymphoma}

Zhaoming Li 10 1,2,9, Xudong Zhang 1,2,9, Weili Xue ${ }^{1,3,9}$, Yanjie Zhang ${ }^{1,3,9}$, Chaoping Li ${ }^{1,3}$, Yue Song ${ }^{1,3}$, Mei Mei ${ }^{1,3}$, Lisha Lu1,3, Yingjun Wang ${ }^{1,3}$, Zhiyuan Zhou1,3, Mengyuan Jin 1,3, Yangyang Bian ${ }^{4}$, Lei Zhang1,2, Xinhua Wang ${ }^{1,2}$, Ling $\mathrm{Li}^{1,2}$, Xin $\mathrm{Li}^{1,2}$, Xiaorui $\mathrm{Fu}^{1,2}$, Zhenchang Sun ${ }^{1,2}$, Jingjing $\mathrm{Wu}^{1,2}$, Feifei Nan ${ }^{1,2}, \mathrm{Yu}$ Chang ${ }^{1,2}$, Jiaqin Yan ${ }^{1,2}$, Hui $\mathrm{Yu}^{1,2}$, Xiaoyan Feng ${ }^{1,2}$, Guannan Wang ${ }^{5}$, Dandan Zhang ${ }^{5}$, Xuefei Fu' ${ }^{6}$, Yuan Zhang ${ }^{7}$, Ken H. Young ${ }^{8}$, Wencai $\mathrm{Li}^{5} \&$ Mingzhi Zhang ${ }^{1,2}$

Natural killer/T cell lymphoma (NKTCL) is a rare and aggressive malignancy with a higher prevalence in Asia and South America. However, the molecular genetic mechanisms underlying NKTCL remain unclear. Here, we identify somatic mutations of GNAQ (encoding the T96S alteration of Goq protein) in 8.7\% (11/127) of NKTCL patients, through wholeexome/targeted deep sequencing. Using conditional knockout mice ( Ncri-Cre-Gnaqfl/fl), we demonstrate that $G \alpha q$ deficiency leads to enhanced NK cell survival. We also find that $G \alpha q$ suppresses tumor growth of NKTCL via inhibition of the AKT and MAPK signaling pathways. Moreover, the Goq T96S mutant may act in a dominant negative manner to promote tumor growth in NKTCL. Clinically, patients with GNAQ T96S mutations have inferior survival. Taken together, we identify recurrent somatic GNAQ T96S mutations that may contribute to the pathogenesis of NKTCL. Our work thus has implications for refining our understanding of the genetic mechanisms of NKTCL and for the development of therapies.

\footnotetext{
${ }^{1}$ Department of Oncology, The First Affiliated Hospital of Zhengzhou University, 450052 Zhengzhou, China. ${ }^{2}$ Lymphoma Diagnosis and Treatment Center of Henan Province, 450000 Zhengzhou, China. ${ }^{3}$ Institute of Clinical Medicine, The First Affiliated Hospital of Zhengzhou University, 450052 Zhengzhou, China. ${ }^{4}$ Medical Research Centre, The First Affiliated Hospital of Zhengzhou University, 450052 Zhengzhou, China. ${ }^{5}$ Department of Pathology, The First Affiliated Hospital of Zhengzhou University, 450052 Zhengzhou, China. ${ }^{6}$ Novogene Bioinformatics Technology Co, Ltd, 38 Xueqing Road, 100083 Beijing, China. ${ }^{7}$ The Academy of Medical Science of Zhengzhou University, 450052 Zhengzhou, China. ${ }^{8}$ Department of Hematopathology, The University of Texas MD Anderson Cancer Center, Houston, TX 77030, USA. ${ }^{9}$ These authors contributed equally: Zhaoming Li, Xudong Zhang, Weili Xue, Yanjie Zhang. Correspondence and requests for materials should be addressed to W.L. (email: wencailizzu@126.com) or to M.Z. (email: mingzhi_zhang@126.com)
} 
$\mathrm{N}$ atural killer/T cell lymphoma (NKTCL) is an aggressive subtype of non-Hodgkin's lymphoma that is rare overall but shows a predilection for Asian and South American populations ${ }^{1-5}$. NKTCLs are almost exclusively extranodal involving the nasal and/or paranasal area, with a strong association with Epstein-Barr virus (EBV) infection ${ }^{3,4,6-8}$. Despite a multimodality chemotherapy and radiotherapy treatment approach, the survival of patients with NKTCL is poor ${ }^{9-12}$.

The molecular pathogenesis of NKTCL currently remains elusive $^{13,14}$. In the past decade, however, several oncogenic pathways, including Janus kinase (JAK) — signal transducer and activator of transcription (STAT), mitogen-activated protein kinase (MAPK), AKT, and NF- $\kappa B$ signaling pathways, have been identified in the development of NKTCL by gene expression profiling ${ }^{15-20}$. Recently, DDX3X, STAT3, JAK3, BCOR, and TP53 have been revealed as novel genes mutated in NKTCL by highthroughput sequencing studies ${ }^{21-28}$.

In this study, we sought to identify additional oncogenic drivers and altered pathways that contribute to NKTCL tumorigenesis in 127 patients with NKTCL through whole-exome/ targeted deep sequencing. In addition to frequently mutated genes reported previously, somatic mutations of GNAQ (encoding the T96S alteration of Gaq protein) were identified in $8.7 \%$ $(11 / 127)$ of the patients with NKTCL. Experiments using conditional knockout mice demonstrated that Gaq deficiency enhanced the survival of natural killer (NK) cells. We also found that Gaq suppressed NKTCL tumor growth via inhibition of the AKT and MAPK signaling pathways. Furthermore, the Gaq T96S mutant might act in a dominant negative manner to promote tumor growth in NKTCL. In addition, we observed that patients with GNAQ T96S mutations had inferior survival.

To our knowledge, the present study includes one of the largest series of NKTCL patients ever described and defines in detail the genetic landscape of mutations. In particular, recurrent GNAQ T96S mutations were detected in our NKTCL patients.

\section{Results}

Whole-exome sequencing of NKTCL. Whole-exome sequencing was performed on paired normal and tumor DNA from 28 patients with NKTCL (Supplementary Fig. 1). The demographics and clinical features of the patients are summarized in Supplementary Table 1 . The mean sequencing depth was $84.67 \times$, and a mean of $91.34 \%$ of the target sequence was covered to a depth of at least 20x (Supplementary Table 2). A total of 2642 nonsilent mutations, including 2374 missense, 114 nonsense, 105 splice site, 2 nonstop, and 47 insertion or deletion mutations, were identified (Supplementary Table 3). The somatic nonsilent mutation load per subject varied significantly in NKTCL (mean 94, range $32-265$, Fig. 1a). Sanger sequencing yielded a $92.11 \%(70 / 76)$ validation rate (Supplementary Table 4 ). Next, we analyzed the mutation spectrum of NKTCL to determine whether mutagenic processes are operative in NKTCL. The predominant type of substitution was a $\mathrm{C} \rightarrow \mathrm{T}$ transition at $\mathrm{NpCpG}$ sites in NKTCL (Fig. 1b). Combining the nonnegative matrix factorization clustering and correlation with the 30 curated mutational signatures defined by the catalog of somatic mutations in cancer (COSMIC) database $^{29}$ revealed three predominant signatures in NKTCL (Fig. 1c, d). The predominantly matched signature was Signature 1 (cosine similarity, 0.84), which was found in all tumor types and is thought to result from age-related accumulation of 5methylcytosine deamination events.

Furthermore, we evaluated the relationship between somatic nonsilent mutation burden and the clinical characteristics of NKTCL. The results showed that somatic nonsilent mutation burden was correlated with patient age $\left(R^{2}=0.196, P=0.018\right.$;
Fig. 1e), and a significant correlation remained $\left(R^{2}=0.304, P=\right.$ 0.0035; Supplementary Fig. 2) after excluding two patients with more than 200 mutations. However, no association was found between nonsilent mutation burden and other clinical features, such as gender, stage, and Eastern Cooperative Oncology group (ECOG) score. Moreover, no relationship between somatic nonsilent mutation burden and overall survival (OS) was observed in NKTCL patients revealed according to survival analysis $(P=0.397$; Fig. 1f $)$.

Recurrent GNAQ mutations in NKTCL. Through whole-exome sequencing, frequent mutations in GNAQ, TET2, and USP8, as well as DDX3X and STAT3 genes previously reported, were identified in our cohort of patients with NKTCL. Prompted by this discovery, we performed targeted deep sequencing in an extended validation group of 73 NKTCL cases. A total of 221 genes, including recurrently mutated genes detected by our exome sequencing and other genes previously reported to be mutated in NKTCL, were sequenced (Supplementary Table 5). The mean average coverage of the target genes was $1408 \times$ (a minimum of $1011 \times$ ), and a mean of $99.38 \%$ of the target sequence was covered to a depth of at least $100 \times$ (Supplementary Tables 6 and 7).

To identify the frequently mutated genes in NKTCL, we combined the sequencing data of the discovery cohort and the validation cohort. After excluding implausible genes, such as the genes encoding olfactory receptors and extremely large proteins, and genes with very long introns, we identified the following 14 genes: $D D X 3 X(16 / 101), K M T 2 D$ (also known as MLL2, 13/101), STAT3 (12/101), GNAQ (11/101), FAT1 (10/101), MSN (9/101), ARID1A (9/101), TET2 (8/101), USP8 (6/101), TP53 (6/101), PTPN13 (6/101), CD93 (5/101), ATRX (5/101), and TRAM1L1 (5/ $101)$. Each of these gene mutations was present in at least five NKTCL samples (Fig. 2a).

Notably, 15 point mutations in GNAQ were identified in 11 NKTCL samples: 9 mutations encoding T96S, 4 mutations encoding Y101X, 1 mutation encoding p.K57T, and 1 mutation encoding p.M59L. Mutations in T96S cooccurred with Y101X and p.M59L in three cases and one case, respectively (Fig. $2 \mathrm{~b}$ and Supplementary Fig. 3). The frequently mutated genes were further validated in another independent cohort of NKTCL patients without matched normal tissue by performing whole-exome sequencing ( $n=26$, Supplementary Table 8). Interestingly, GNAQ T96S mutations were found in 2 of the 26 examined cases, and both were subsequently validated by Sanger sequencing (Supplementary Fig. 4). In this study, GNAQ T96S mutations were identified in $8.7 \%(11 / 127)$ of the NKTCL cases.

Gaq deficiency leads to enhanced NK cell survival. NKTCL is a mature NK cell neoplasm ${ }^{30,31}$. However, the function of GNAQ in normal NK cells is unclear. To address this question, we generated Ncr1-Cre-Gnaqfl/fl mice that express Cre recombinase under control of the Ncr1 promoter, which allowed NK cellspecific ablation of Gnaq (Fig. 3a, b). Ncr1-Cre-Gnaqfl/fl mice were born at the expected Mendelian ratio without any visible alterations in organ morphology or overt pathology.

First, we verified Gaq expression in sorted NK cells isolated from Ncr1-Cre-Gnaqflfl mice and their littermate controls that harbored loxP-flanked Gnaq alleles but lacked Ncr1-Cre (Gnaqfl/fl mice). As expected, splenic NK cells from Ncr1-Cre-Gnaqflfl mice showed significantly reduced Gaq expression compared with NK cells from control mice; however, there was no difference in Gaq expression on non-NK cells between the two groups (Fig. 3c). Therefore, Gnaq was deleted exclusively in NK cells. 

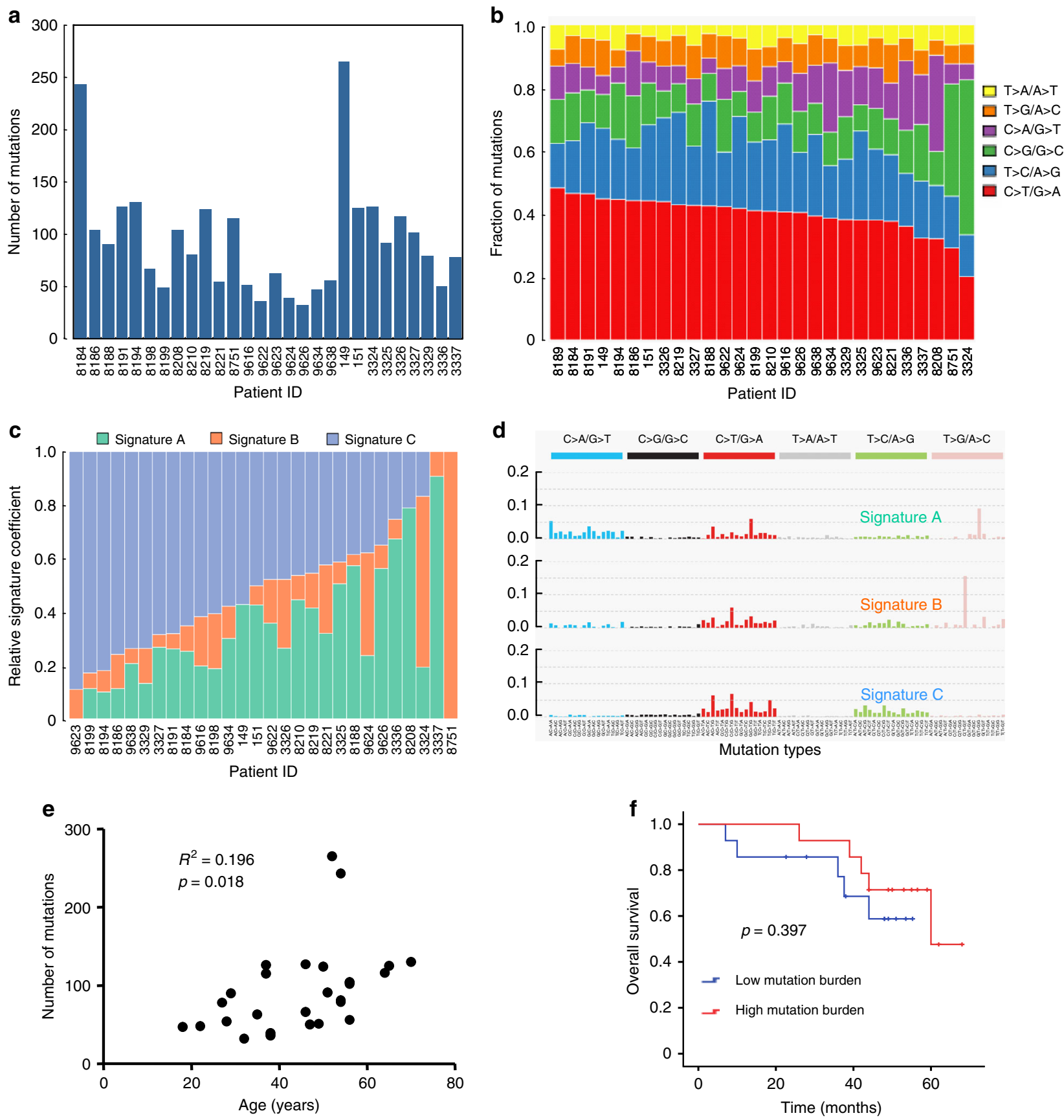

Fig. 1 Whole-exome sequencing in 28 patients with NKTCL. a The number and type of nonsilent somatic mutations identified by whole-exome sequencing. b The spectrum of mutations in NKTCL. c, $\mathbf{d}$ Three dominant signatures identified by combined nonnegative matrix factorization clustering and correlation in NKTCL, with 30 curated mutational signatures defined by the COSMIC database. $\mathbf{e}$ The correlation analysis of nonsilent somatic mutations and the age of the NKTCL patients $\left(n=28 ; R^{2}=0.196, P=0.018\right)$. $\mathbf{f}$ The association of the somatic nonsilent mutation burden with overall survival in NKTCL (log-rank $P=0.397)$

Next, we determined whether Gnaq influences the distribution of NK cells within different compartments. However, no significant differences in NK cell frequency were detected among tissue types (bone marrow, spleen, peripheral blood, liver, and lymphoma node) in Ncrl-Cre-Gnaqfl/fl mice and their littermate controls (Fig. 3d).

Finally, we evaluated whether Gnaq is involved in the regulation of NK cell expansion or survival. The expansion of splenic NK cells was assessed by an in vivo BrdU incorporation assay. No differences in DNA replication were observed in splenic NK cells between Ncr1-Cre-Gnaq $q^{l / f l}$ mice and their littermate controls (Fig. 3e). We further investigated whether Gnaq regulates NK cell survival. Purified splenic NK cells were cultured in media without IL-2 for $6 \mathrm{~h}$, and cell viability was monitored by Annexin V staining. Surprisingly, NK cells from Ncr1-Cre-Gnaq fl/ $f l$ mice exhibited a significant survival advantage compared with NK cells from their corresponding littermate controls (Fig. 3f). Collectively, these data suggested that NK cells from mice lacking Gnaq have an intrinsic survival advantage over normal NK cells.

Tumor-suppressive role of Gaq in NKTCL. We next sought to determine the functional role of Gaq in NKTCL. First, we examined the expression of GNAQ in NK cells isolated from 

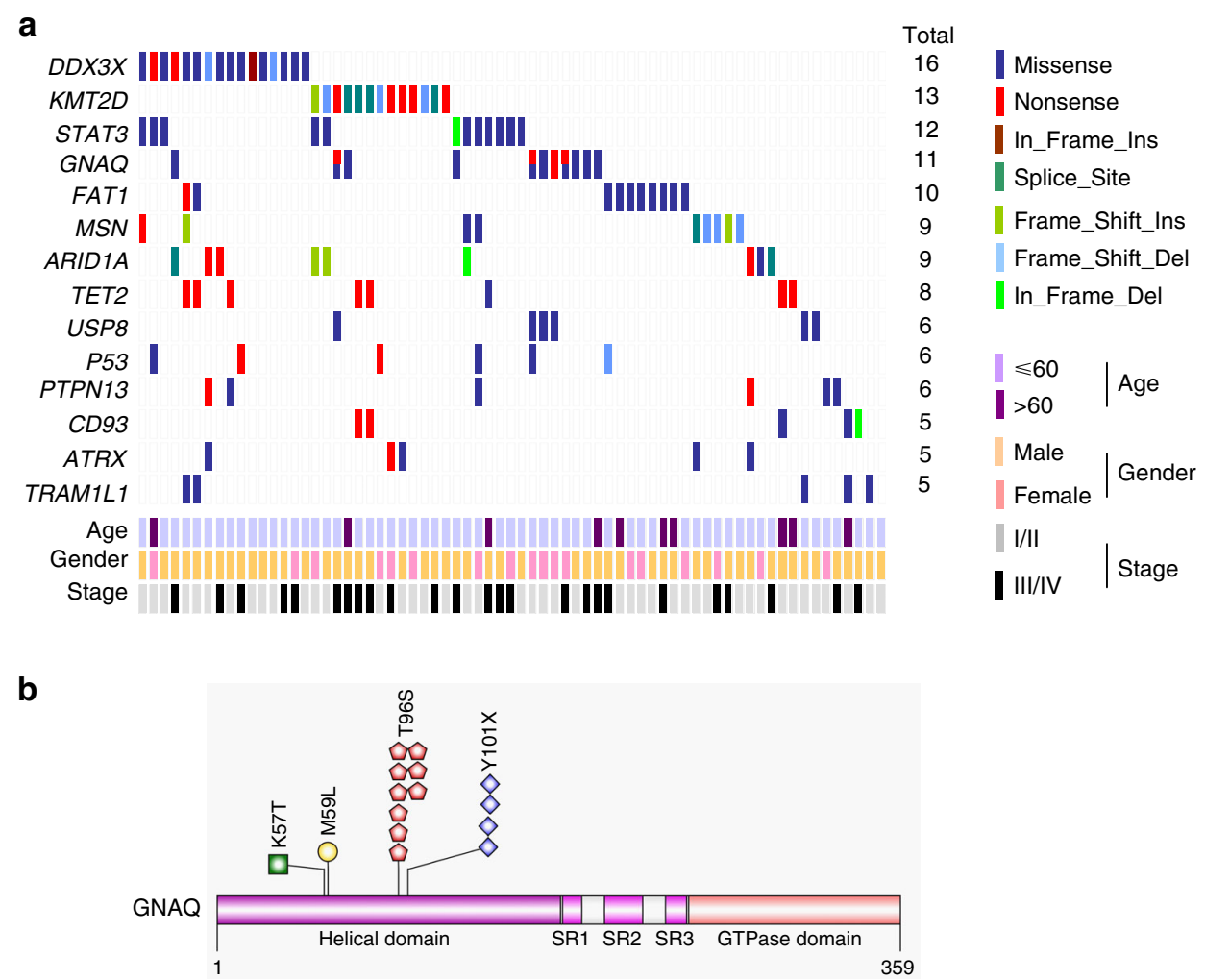

Fig. 2 Frequently mutated genes. a Fourteen frequently mutated genes are ranked according to the frequency of mutations. Samples are displayed in columns. Gene mutations are shown in different colors according to the type of alteration. The total number of patients with mutations in each gene is listed on the right. b Mapping of GNAQ mutation sites in the total NKTCL patient cohort. Functional domains of the altered proteins are based on the UniProt database

normal and neoplastic tissues. A pooled analysis of previously published datasets ${ }^{15}$ and our own RNA sequencing data showed that GNAQ expression was significantly lower in neoplastic NK cell lines and tumor samples compared with primary human NK cells (Fig. 4a). This result was further confirmed by western blot analysis of Gaq protein expression in primary human NK cells and different neoplastic NK cell lines. In particular, loss of Gaq expression was observed in a novel NK leukemia cell line (KHYG1) (Fig. 4b). Chromosome 9q deletion has been reported in KHYG1, which may account for the loss of Gaq in this cell line ${ }^{32,33}$. Additionally, somatic copy number alteration analysis was performed in the 28 exome cases, and somatic copy number loss of GNAQ was found in one case (Supplementary Fig. 5).

Second, we generated stable cell lines (YT and NKYS) expressing vector control or GNAQ. Both cell lines carried wild-type GNAQ according to the RNA sequencing data ${ }^{15}$. As shown in Fig. 4c, forced expression of Gaq significantly suppressed tumor cell viability in comparison with the vector control in both cell lines. We further examined the effect of Gaq on cell apoptosis and proliferation by Annexin $\mathrm{V}$ staining and EdU incorporation assay, respectively. In vitro studies demonstrated that overexpression of Gaq significantly enhanced cell apoptosis but had little effect on NK cell proliferation (Fig. 4d).

Finally, YT cells stably expressing Gaq or vector control cells were injected subcutaneously into two groups of NOD/SCID mice ( $n=5$ each). The tumor size was measured every 2 days with calipers, and tumor burden was monitored 6 weeks after cell implantation. As expected, in vivo fluorescence imaging of xenograft mice showed that the fluorescence intensity $\left(\mathrm{p}^{\wedge}\right.$ $\left.1 \mathrm{sec}^{\wedge}-1 \mathrm{~cm}^{\wedge}-2 \mathrm{sr}^{\wedge}-1\right)$ of tumors derived from the Gaq group was significantly lower than those from the control group $(P=0.011$; Fig. 4e). Consistently, the mean tumor weight of the Gaq group at the end of the experiment was significantly lower $(0.81 \pm 0.24 \mathrm{~g})$ than that of the control group $(0.21 \pm 0.12 \mathrm{~g}, P=0.002$; Fig. $4 \mathrm{e})$. We further addressed the question of whether Gaq affected cell survival in vivo. The number of apoptotic cells was significantly higher in the Gaq group than in the vector control group, as determined by the TDT-mediated dUTP nick end labeling (TUNEL) assay ( $P=0.031$; Fig. $4 \mathrm{f})$. In addition, the YT cells expressing Gaq were significantly less proliferative based on Ki67 staining and analysis of tumor sections (Supplementary Fig. 6). This finding indicated that overexpression of Gaq affects the survival and/or proliferative capacity of YT cells in the tumor microenvironment, which appears to be the mechanism by which Gaq can suppress tumor growth in vivo.

Gaq T96S mutants act in a dominant negative manner in NKTCL. We then attempted to characterize the functional role of the Gaq T96S mutant in NKTCL. Because all Gaq mutationpositive cases of NKTCL were heterozygous for this mutation, we established tetracycline-inducible cell lines (KHYG1 and YT) that could simultaneously express equal levels of wild-type and mutant Gaq. The expression levels of wild-type and Gaq T96S mutant were verified by western blot (Fig. 5a).

Expression of wild-type Gaq led to a significant decrease in the viability of NK cells. In contrast, the expression of the Gaq T96S mutant could compromise the ability of wild-type Gaq to impair NK cell viability (Fig. 5b). However, no significant difference between the Gaq T96S mutant and control groups was present, possibly due to the absent or extremely low expression of endogenous Gaq in these two NK cell lines. Similarly, forced expression of wild-type Gaq enhanced cell apoptosis of NK cells, and this proapoptotic effect of wild-type Gaq could be attenuated by the Gaq T96S mutant (Fig. 5c), suggesting a dominant 
a

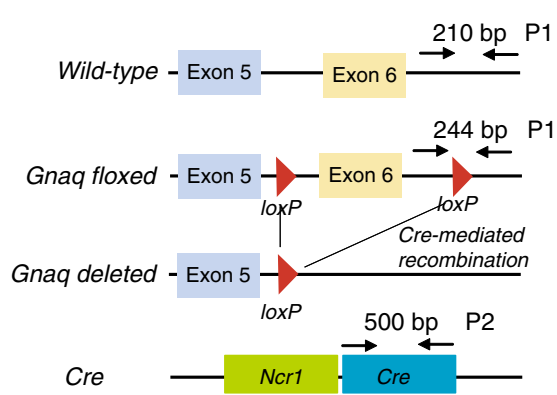

C
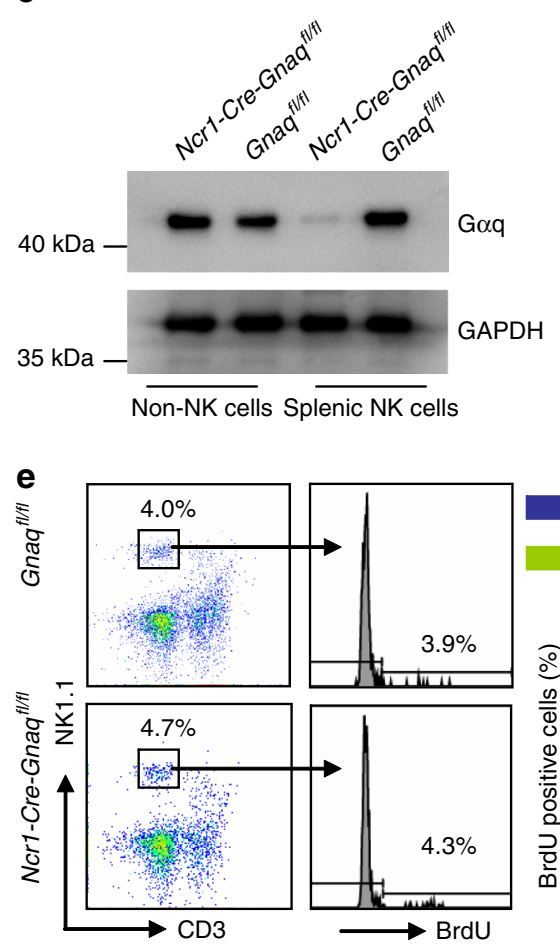

b

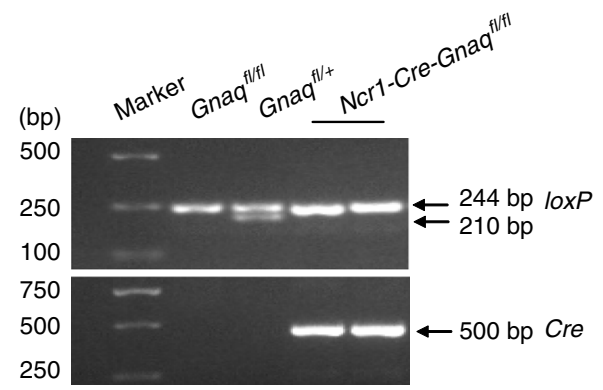

d
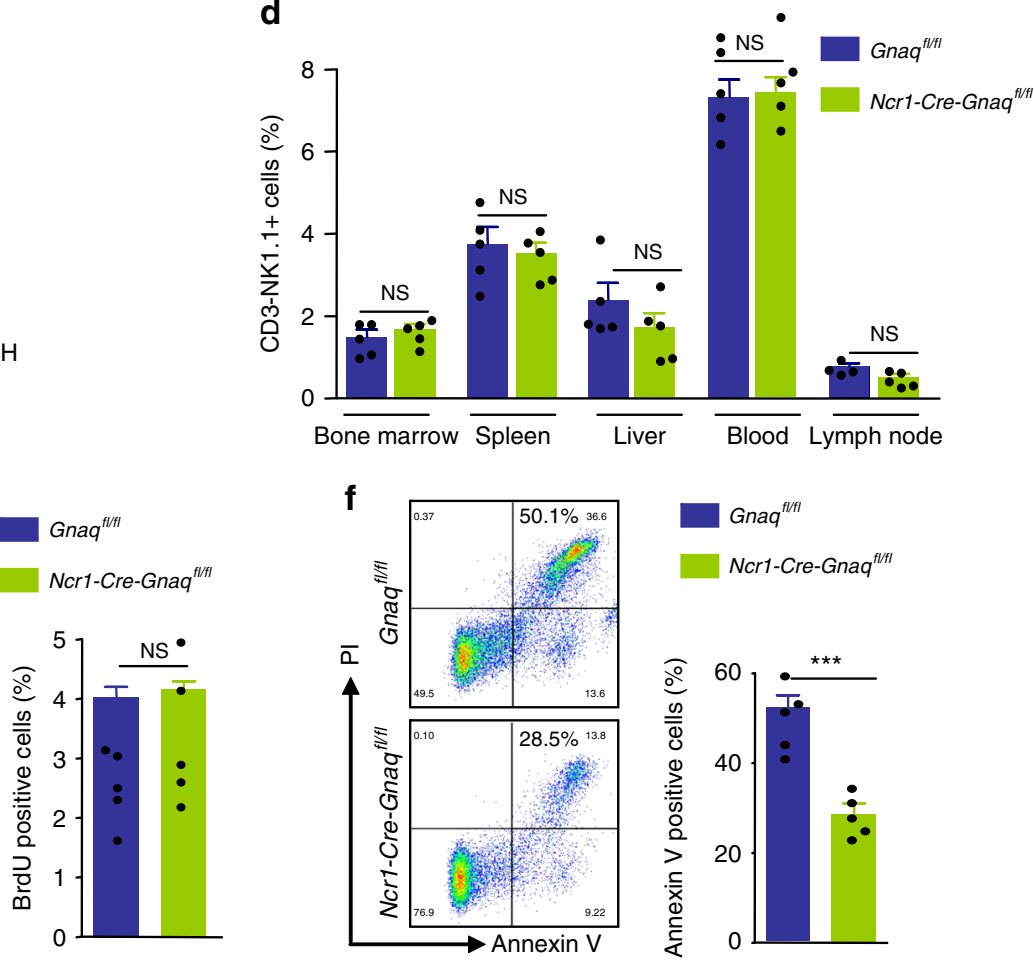

f

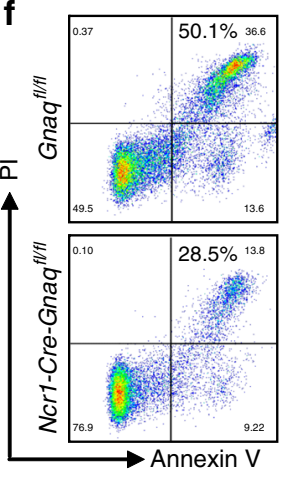

Gnaq $^{f / f t}$ Ncr1-Cre-Gnaq ${ }^{\text {tht }}$

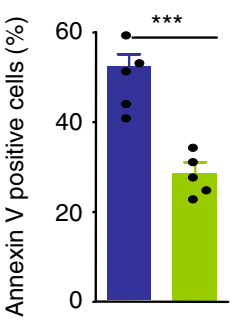

Fig. 3 Gaq deficiency leads to enhanced NK cell survival. a In Gnaq ${ }^{f l / f l}$ mice, exon 6 of the Gnaq gene is flanked with loxP sites. Crossing Gnaq ${ }^{f l / f l}$ mice with Ncri-Cre mice leads to Cre-mediated recombination, resulting in exon 6 deletion. The diagram is not to scale. $\mathbf{b}$ Genotyping of mouse tail DNA for the presence of Cre recombinase transgene and a floxed transgene. Genomic PCR was performed on DNA derived from different mice. The presence of the Cre recombinase gene is detected by the amplification of a 500-bp product. The floxed products expected (244 bp) are shown. c Goq protein levels were measured by western blot in splentic NK and non-NK cells from Ncr7-Cre-Gnaq $q^{f l / f l}$ and Gnaq $q^{f / f}$ mice. $\mathbf{d}$ The frequencies of NK cells in the bone marrow, spleen, peripheral blood, liver, and lymph node in Ncr1-Cre-Gnaqfl/fl and Gnaqfl/f mice ( $n=5$ per genotype). Bar graphs show the percentage of NK cells $\left(\mathrm{CD} 3^{-} \mathrm{NK} 1.1^{+}\right)$. Data are representative of two independent experiments. e In vivo proliferation of splenic NK cells. Mice were injected intraperitoneally with BrdU ( $2 \mathrm{mg}$ ), and after $12 \mathrm{~h}$, the incorporation of BrdU in splenic NK cells was analyzed. Numbers adjacent to outlined areas in the dot plot indicate the percentage of $\mathrm{CD}^{-}{ }^{-} \mathrm{NK} 1.1^{+}$cells. Histograms show the percentage of BrdU-positive cells ( $n=5$ per genotype). Data are representative of two independent experiments. f Purified splenic NK cells were cultured in media without IL-2 for $6 \mathrm{~h}$, and viability was monitored by Annexin $V$ staining. Histograms show the percentage of Annexin $\vee$-positive cells ( $n=5$ per genotype). Data are representative of two independent experiments. All data are expressed as the mean \pm s.e.m.; NS, not significant. ${ }^{\star} P<0.05,{ }^{\star \star} P<0.01$, ${ }^{\star \star \star} P<0.001$, unpaired two-tailed Student's $t$-test. Source data are provided as a Source Data file

negative nature of Gaq T96S. Moreover, we examined the effect of the Gaq T96S mutant on NKTCL tumorigenesis in vivo. Consistent with the above findings, wild-type Gaq significantly reduced the tumor-formation ability compared with the vector control, whereas the Gaq T96S mutant impaired the tumorsuppressor activity of wild-type Gaq. The mean weights of the tumors in the vector control, wild-type, Gaq T96S, and wildtype + Gaq T96S groups were $(0.46 \pm 0.08),(0.09 \pm 0.02),(0.41 \pm$ $0.06)$, and $(0.37 \pm 0.07) \mathrm{g}$, respectively (wild-type versus vector, $P=0.002$; Gaq T96S versus vector, not significant; Fig. 5d).

Furthermore, we identified protein binding partners of wildtype or Gaq T96S mutants using immunoprecipitation coupled to mass spectrometry. Significance analysis of INTeractome (SAINT) expression was utilized to calculate the probability of protein-protein interaction from background, nonspecific interactions ${ }^{34-37}$. Using a SAINT probability threshold of $\geq 0.8$, we identified 17 high-confidence interacting partners of Gaq (Fig. 5e and Supplementary Table 9). Gß1 (GNB1), a known direct binding partner of Gaq, appeared to have higher affinity for the Gaq T96S mutant than its wild-type. Furthermore, immunoprecipitation assay confirmed that the Gaq T96S mutant bound G $\beta 1$ more tightly than wild-type Gaq in YT cells (Fig. 5f). It is well known that the Ga mutant can exert a dominant negative effect in the heterotrimeric complex 

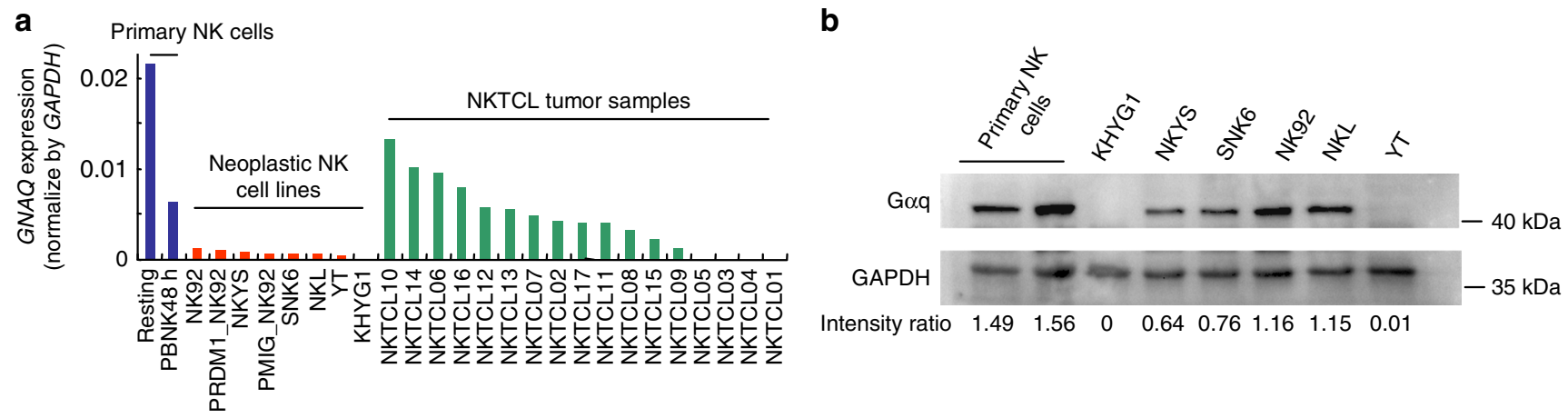

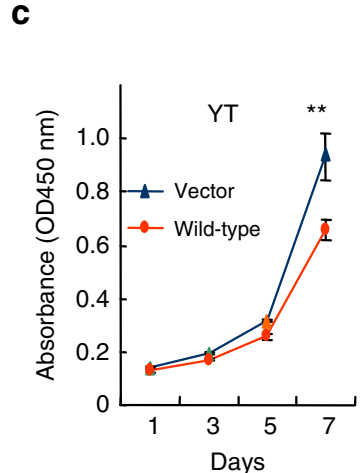

e

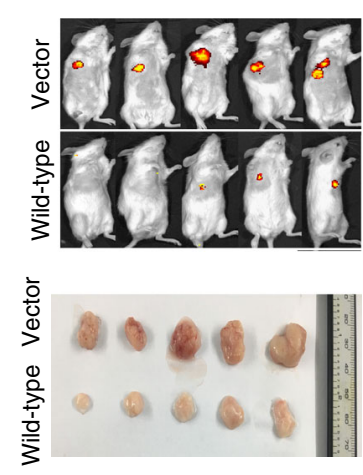

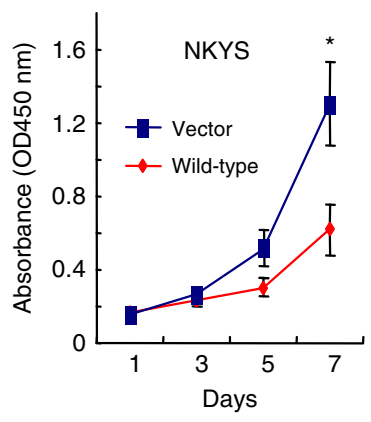
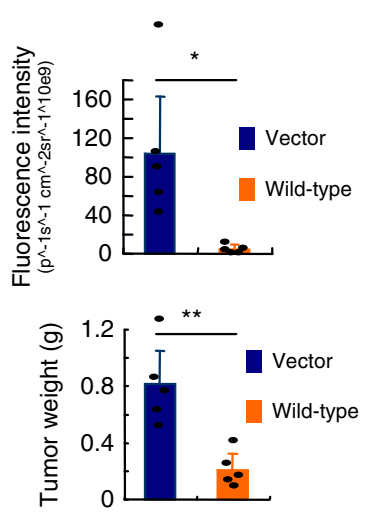

d
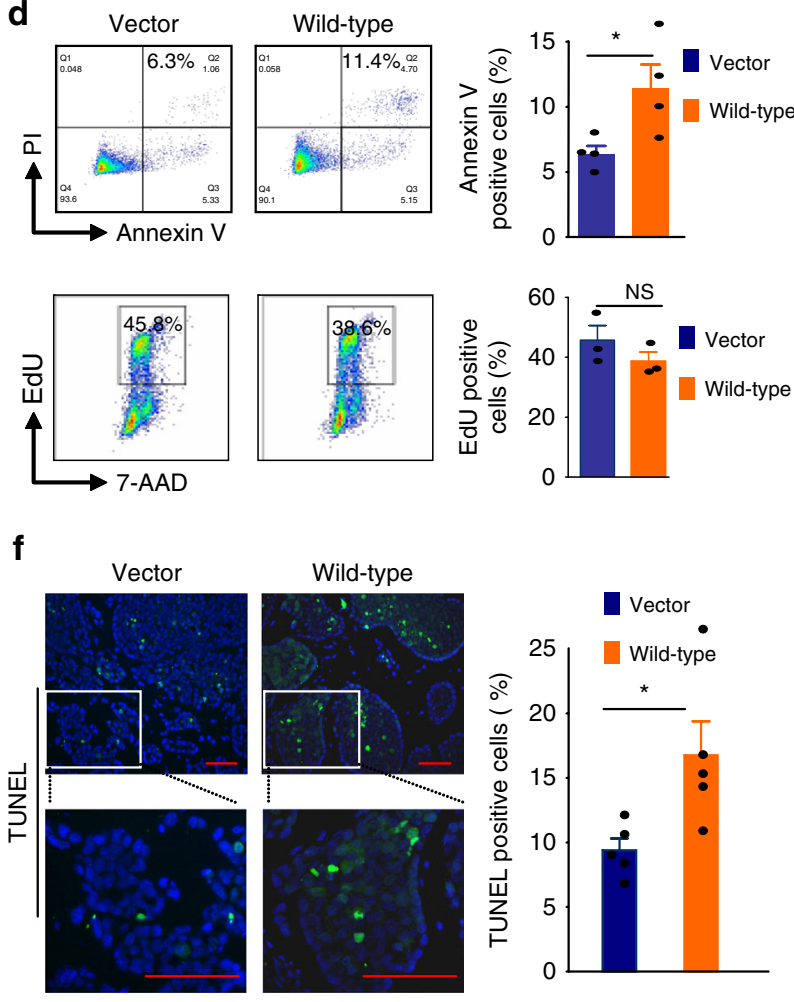

Fig. 4 Tumor-suppressive role of G $\alpha$ in NKTCL. a GNAQ mRNA expression in normal NK cells, neoplastic NK cells, and tumor samples. The GNAQ expression values were obtained from previously published data and our RNA sequencing data and normalized to GAPDH. Resting NK: >95\% CD56+CD3NK cells isolated from peripheral blood lymphocytes. PBNK48h: 48-h IL-2-activated NK cells. PMIG_NK92 and PRDM1_NK92: NK92 cells transduced with PMIG and PRDM1, respectively. b GNAQ protein expression in normal and neoplastic NK cells. c Forced expression of Goq suppressed cell viability in YT (left) and NKYS (right) cells. YT and NKYS cells were stably transfected with vector control or wild-type GNAQ, and cell viability was assessed using a CCK-8 assay. Cell viability is presented by the absorbance value at OD $450 \mathrm{~nm}$, which was measured with a Multiskan FC microplate reader (Thermo Scientific, Waltham, MA, USA). The value is directly proportional to the number of viable cells in the culture medium. Data are representative of three independent experiments. d Overexpression of Goq significantly enhanced cell apoptosis but had little effect on NK cell proliferation. Cell apoptosis and cell proliferation were assessed by Annexin $\mathrm{V}$ staining (upper panel) and EdU incorporation assay (lower panel), respectively. Data are representative of at least three independent experiments. e NOD/SCID mice were inoculated subcutaneously with YT cells stably transfected with vector control or wild-type Goq ( $n=5$ in each group). The tumor burden was monitored by utilizing the IVIS Spectrum system (Perkin Elmer, Beaconsfield, UK) after 6 weeks. Representative images and quantitative data for each group are shown in the upper panel. Representative images of xenograft tumors and tumor weights for each group are shown in the lower panel. $\mathbf{f}$ Representative images (left) and quantitative data (right) ( $n=5$ in each group) for TUNEL staining of the xenograft tumor tissues. Scale bars, $50 \mu \mathrm{m}$. All data are expressed as the mean \pm s.e.m.; NS, not significant. ${ }^{\star} P<0.05$, ${ }^{\star \star} P<0.01,{ }^{\star \star \star} P<0.001$, unpaired two-tailed Student's $t$-test. Source data are provided as a Source Data file

by sequestration of the $G \beta \gamma$ subunits ${ }^{38}$. It is therefore suggested that sequestering the $\mathrm{G} \beta$ subunits may be a possible mechanism by which the Gaq T96S mutant exerts its dominant negative effect on wild-type Gaq in NK cells, which should be further investigated. Altogether, these data suggested that the Gaq T96S mutant might contribute to the pathogenesis of NKTCL through the inhibition of wild-type Gaq in a dominant negative manner.
Gaq suppresses AKT and MAPK signaling pathways in NK cells. To examine the effect of Gaq on gene expression in NK cells, we performed RNA sequencing analysis using RNA prepared from NKYS cells stably expressing vector control or wildtype Gaq. Gene set enrichment analysis (GSEA) demonstrated that both MAPK and T cell receptor (TCR) signaling pathways were significantly enriched in cells expressing the vector control compared with wild-type Gaq cells (Fig. 6a and Supplementary 
a

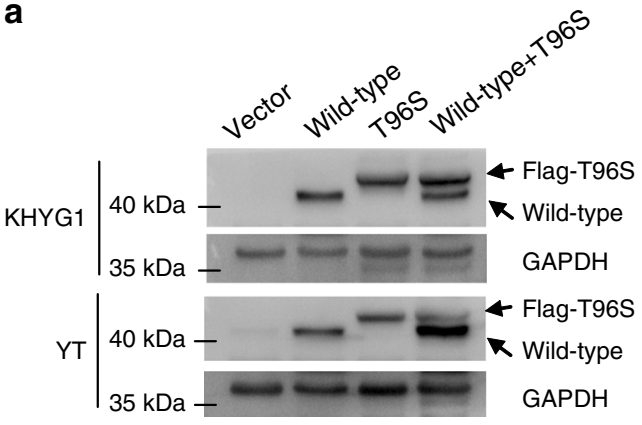

b

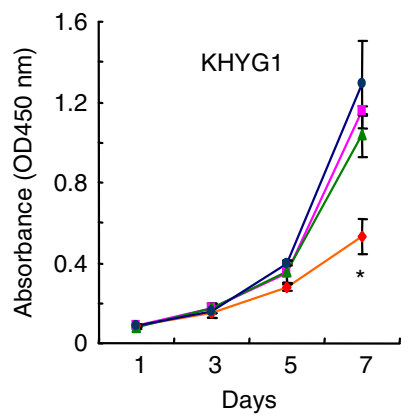

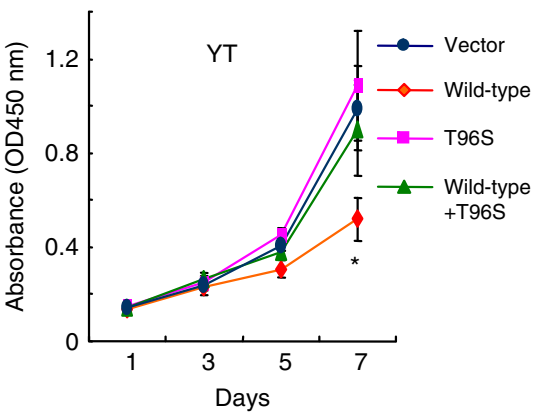

C

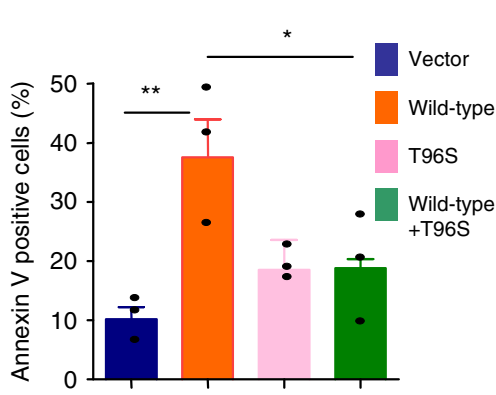

d
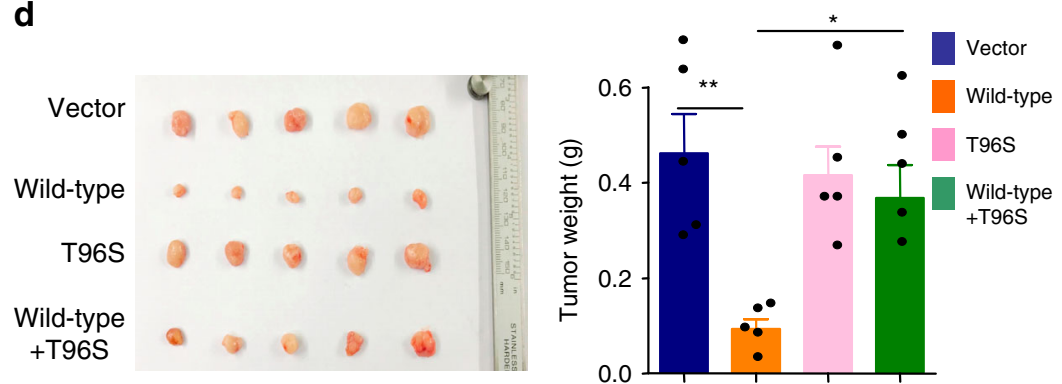

f

e

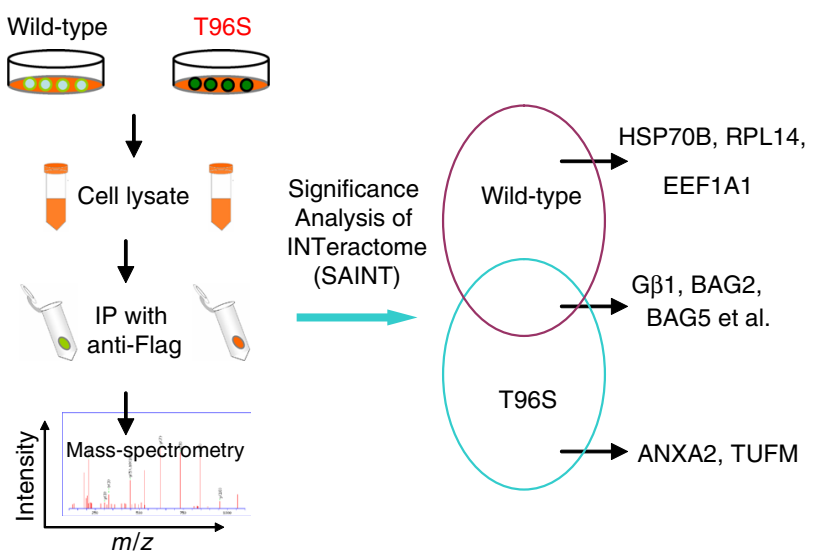

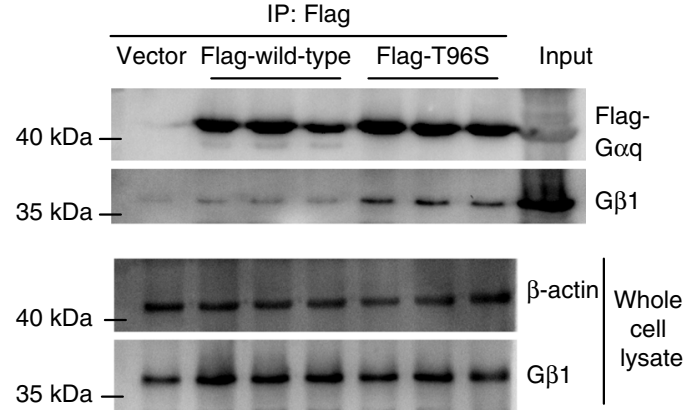

Fig. 5 G $\alpha$ T96S mutant acts in a dominant negative manner to promote tumor growth of NKTCL. a Western blot shows that tetracycline-inducible cell lines (KHYG1 and YT) express equal amounts of wild-type and mutant Goq simultaneously. b Viability of YT and KHYG1 cells with inducible expression of vector, wild-type, T96S or wild-type plus T96S. Cell viability is presented as the absorbance value at OD $450 \mathrm{~nm}$. Data are representative of three independent experiments. c Apoptosis was induced after starving KHYG1 cells of IL-2 for $12 \mathrm{~h}$ and was assessed by Annexin $\mathrm{V}$ staining assay. Data are representative of three independent experiments. $\mathbf{d}$ Representative images of xenograft tumors (left) and tumor weights (right) for YT cells with inducible expression of vector, wild-type, T96S or wild-type plus T96S ( $n=5$ in each group). e Schematic diagram of the approach used to identify G $\alpha q$-interacting partners using immunoprecipitation in combination with mass spectrometry. Significance analysis of INTeractome (SAINT) expression was utilized to calculate the probability of protein-protein interactions from background, nonspecific interactions. The right panel shows representative binding proteins

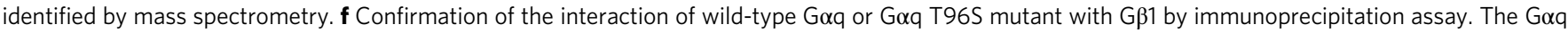
T96S mutant bound G $\beta 1$ more tightly than wild-type G $\alpha q$ in $Y T$ cells. All data are expressed as the mean \pm s.e.m.; NS, not significant. ${ }^{\star} P<0.05,{ }^{\star \star} P<0.01$, ${ }^{\star \star *} P<0.001$, unpaired two-tailed Student's $t$-test. Source data are provided as a Source Data file

Table 10). Consistently, NK cells from Ncr1-Cre-GNAQ ${ }^{f l / f l}$ mice showed significantly higher expression of phospho-ERK (p-ERK) and phospho-AKT (p-AKT) than their control littermates (Fig. 6b). These results indicated that Gaq could suppress AKT and MAPK signaling pathways in NK cells, consistent with previous studies reporting that these two pathways could be suppressed by Gaq in mice B and T cells ${ }^{39,40}$.

These findings were further validated in NKTCL cells. As shown in Fig. 6c, compared with the vector control group, the phosphorylation of ERK and AKT was suppressed in the presence of wild-type Gaq. However, unlike wild-type Gaq, the Gaq T96S mutant was unable to reduce the phosphorylation of ERK and AKT. In contrast, it could affect the suppressive function of wild-type Gaq on the two signaling pathways, underlining a dominant negative nature of Gaq T96S. Similar results were obtained from immunohistochemistry analysis of mouse xenograft tumors (Fig. 6d). Moreover, phospho-ERK and phospho-AKT were detected in human NKTCL samples, with significantly higher levels in patients with the mutant GNAQ T96S genotype than in patients with the wild-type Gaq genotype using two immunohistochemistry scoring methods (Fig. 6e and Supplementary Fig. 7).

GNAQ T96S mutations predict a worse prognosis. We investigated the relationship between GNAQ T96S mutations and clinical characteristics. In this analysis, only patients with 

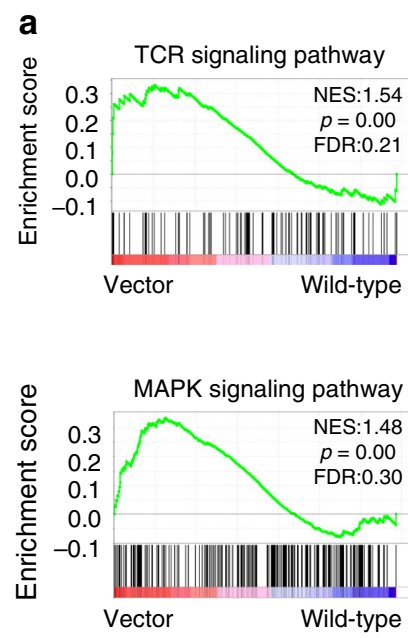

b

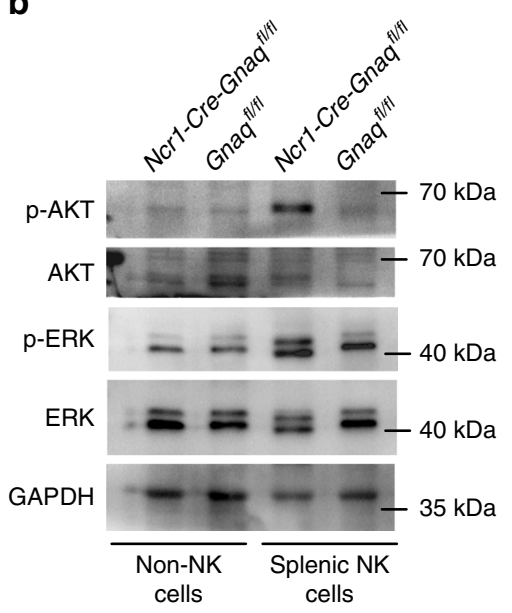

c

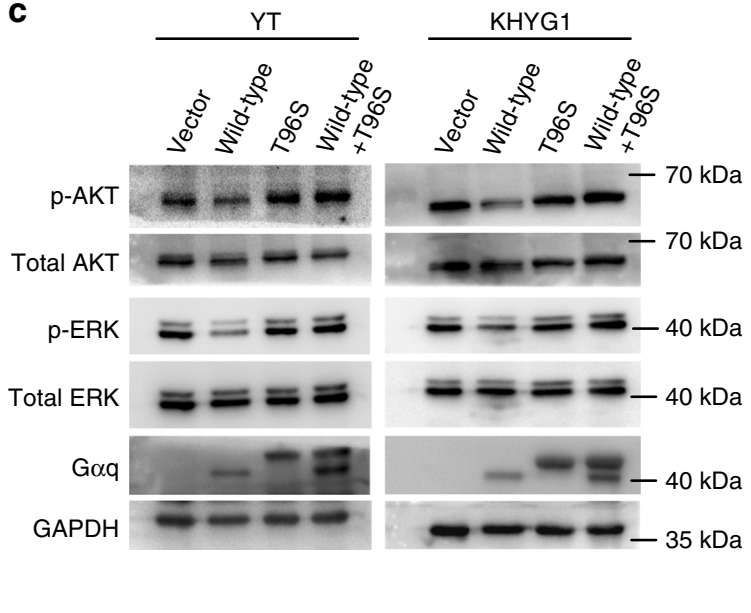

d

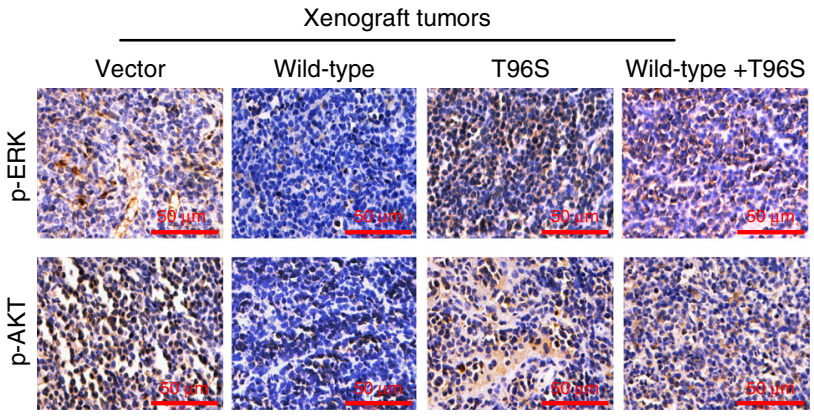

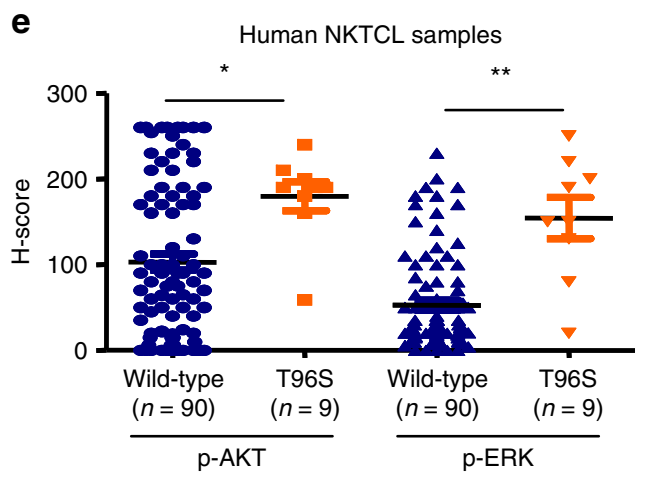

Fig. $6 \mathrm{G} \alpha q$ suppresses AKT and MAPK signaling pathways in NK cells. a RNA sequencing and subsequent gene set enrichment analysis (GSEA) were performed on NKYS cells stably expressing vector control or wild-type Gaq. The P value of GSEA was computed by a 1000-gene-set two-sided permutation test. b p-ERK and p-AKT expression in NK cells from Ncr1-Cre-Gnaq $q^{f l / f l}$ and Gnaq ${ }^{f l / f l}$ mice. c The effect of wild-type Gaq or T96S mutant on the activation of ERK and AKT in YT (left) and KHYG1 (right) cells. GAPDH was used as a loading control. d Representative immunostaining of p-ERK and p-AKT in the xenograft tumor tissues; scale bars, $50 \mu \mathrm{m}$. e Immunohistochemical study of p-ERK and p-AKT in human NKTCL samples with wild-type Goq $(n=90)$ and T96S mutant $(n=9)$. The expression levels of p-ERK and p-AKT were scored using the semiquantitative $H$-score (histochemical score) visual approach, taking into consideration the intensity of staining and the percentage of stained cells. All data are expressed as the mean \pm s.e.m.; NS, not significant. ${ }^{\star} P<0.05,{ }^{\star \star} P<0.01,{ }^{\star \star \star} P<0.001$, unpaired two-tailed Student's $t$-test. Source data are provided as a Source Data file

matched tumor/normal sample pairs were included, two individuals with non-T96S GNAQ mutations (Y101X in \#8188 and $\mathrm{K} 57 \mathrm{~T}$ in \#36) were excluded from the survival analysis. We found that GNAQ T96S mutations were significantly associated with advanced disease stage $(P=0.025)$ and a higher fraction of Ki67positive cells $(>50 \%)$ in the tumor samples $(P=0.035$; Supplementary Table 11).

Univariate analysis suggested that patients with GNAQ T96S mutations $(n=9)$ had a worse prognosis than individuals with wild-type GNAQ $(n=90)$. The estimated 3-year OS rates of patients with GNAQ T96S mutations and wild-type GNAQ were $33.3 \pm 15.7 \%$ and $72.3 \pm 5.0 \%$, respectively $(P=0.006, \mathrm{FDR}=$ 0.048; Fig. 7a). The estimated 3-year progression-free survival (PFS) rates for subjects from the GNAQ T96S and wild-type GNAQ groups were $22.2 \pm 13.9 \%$ and $57.3 \pm 6.2 \%$, respectively $(P=0.005, \quad F D R=0.059$; Fig. $7 b)$. Moreover, when a Cox multivariate regression model was applied, the T96S mutation status of GNAQ remained an independent prognostic marker for poor outcomes after adjusting for B symptoms, the International Prognostic Index (IPI), the Ki67 index $(>50 \%)$, and the primary site of the tumor (OS: $\mathrm{HR}=3.31, \mathrm{CI}=1.33-8.24, P=0.010$; and PFS: $\mathrm{HR}=2.85, \mathrm{CI}=1.27-6.37, P=0.011$; Supplementary Table 12). Altogether, these results suggested that GNAQ T96S mutations were positively correlated with advanced tumor stage and poor clinical outcomes in NKTCL.
In addition, mutations in $D D X 3 X$ or TP53 were associated with a worse prognosis of patients with NKTCL, consistent with previous studies ${ }^{41}$. In contrast, patients without mutations in DDX3X, TP53, or GNAQ (T96S) showed a more favorable prognosis than those harboring the mutations (Fig. 7c, d). These observations indicated that the mutational status of $D D X 3 X$, TP53 and GNAQ genes might represent a promising marker for therapeutic stratification of patients with NKTCL, which needs to be validated in future large, prospective studies.

\section{Discussion}

NKTCL is a rare, malignant neoplasm characterized by a highly aggressive clinical course ${ }^{13,42-45}$. Although considerable advances in our understanding of the mechanisms involved in NKTCL have been made in recent years, the rare nature of NKTCL and its heterogeneity limit the ability to standardize therapy ${ }^{13,42-46}$. Somatic mutation analysis represents a useful tool in selecting personalized therapy ${ }^{14,22,47}$. In this study, we undertook largescale screening in a cohort of NKTCL patients and identified recurrent somatic GNAQ mutations encoding a p. T96S alteration in $8.7 \%(11 / 127)$ of NKTCL samples. In addition to GNAQ, mutations in multiple genes involved in $G$ protein-coupled receptor (GPCR) signaling pathways, including GPR98, GPR125, GNAS, GNA12, GNAI2, and PLCB3, were also detected in our 

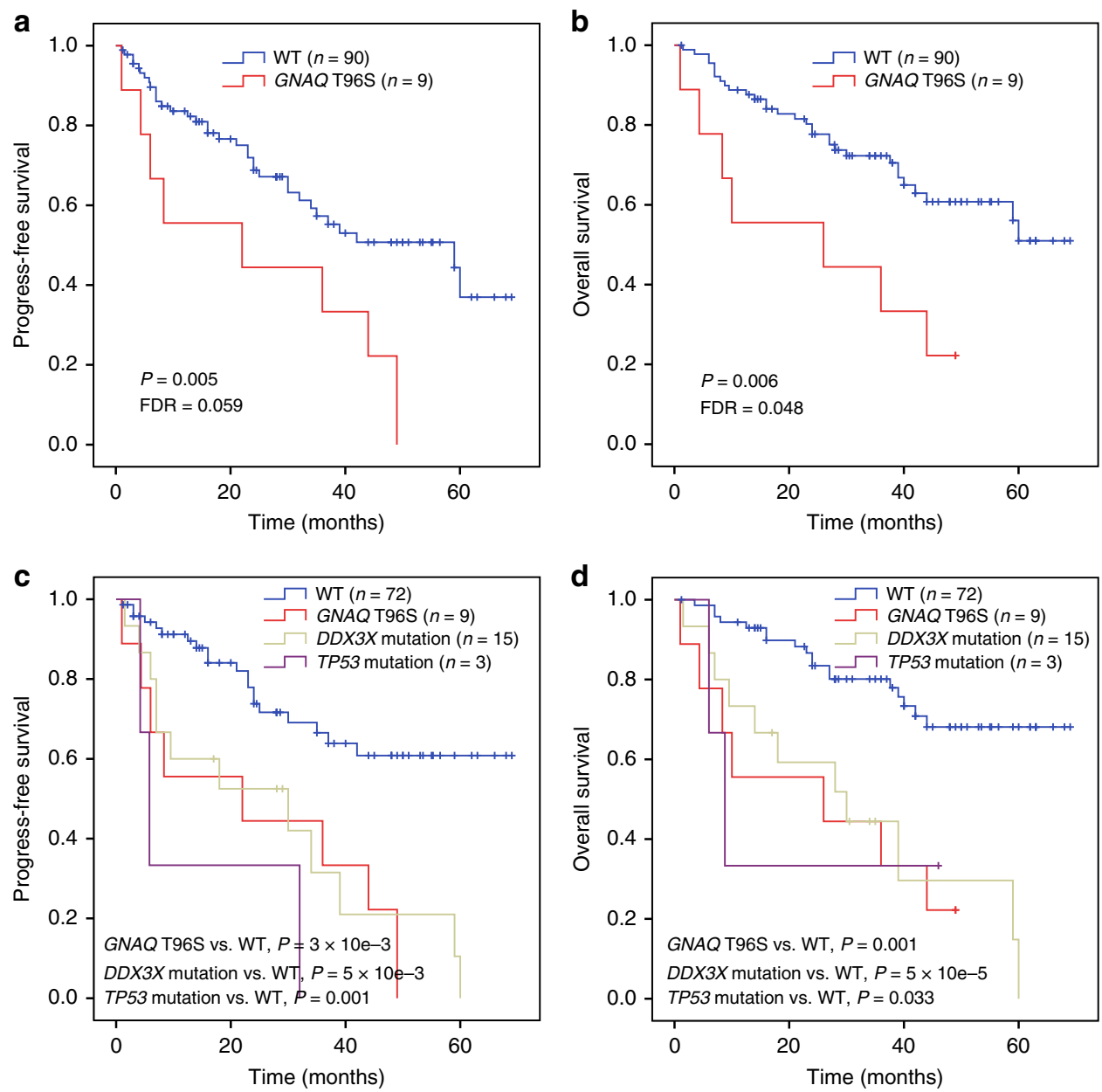

Fig. 7 GNAQ T96S mutations predict a worse prognosis in NKTCL. a, b Progression-free survival (a) and overall survival (b) of NKTCL patients with GNAQ T96S mutations $(n=9)$ or wild-type (WT, $n=90)$. c, $\mathbf{d}$ Progression-free survival (c) and overall survival (d) of patients according to the mutation status of DDX3X, GNAQ, and TP53. Patients were divided into four groups: those with GNAQ T96S mutations $(n=9), D D X 3 X$ mutations ( $n=15)$, or TP53 mutations $(n=3)$ and those without mutations in any of these three genes (WT, $n=72$ ). Two individuals with both DDX3X and TP53 mutations were grouped into the DDX3X cohort. One individual with both GNAQ T96S and TP53 mutations was grouped into the GNAQ T96S cohort. Survival curves were estimated with the Kaplan-Meier method and compared using a two-sided log-rank test

study. These mutations appeared to be mutually exclusive and affected $24.8 \%(25 / 101)$ of total NKTCL cases (Supplementary Fig. 8), suggesting that deregulated GPCR signaling plays a major role in the tumorigenesis of NKTCL.

$\mathrm{Gaq}$ is a member of the $\mathrm{q}$ class of $\mathrm{Ga}$ subunits that mediates signals between GPCRs and downstream effectors ${ }^{48,49}$. Although the oncogenic potential of Gaq has been widely studied in human tumors, its roles in NK cells remain unclear ${ }^{49}$. Using newly generated conditional knockout mice (Ncr1-Cre-GNAQ $\left.{ }^{f l f l}\right)$, we found that NK cells lacking GNAQ have an intrinsic survival advantage over normal counterparts, which is consistent with previous studies in B and T lymphocytes ${ }^{39,40}$. Further functional studies in neoplastic NK cells revealed a tumor-suppressive role of Gaq in NKTCL. Gaq suppressed the activation of AKT and ERK in the same way it did in B and $\mathrm{T}$ lymphocytes ${ }^{39,40}$. Therefore, the dual function of Gaq in different types of tumors suggests that it may potentially act either as a tumor suppressor or as an oncogene depending on the cellular context. This type of functional duality based on cellular context has been observed for Ga13 (encoded by GNA13), which is another member of the Ga protein family ${ }^{49,50}$. Ga13 has been previously linked to cellular transformation and characterized as growth promoting in epithelial cancer models ${ }^{51,52}$. However, recent studies have identified a Ga13-dependent pathway that exerts dual actions in suppressing growth and blocking dissemination of germinal center $\mathrm{B}$ cells, which are frequently disrupted in germinal center B-cellderived lymphoma ${ }^{53-55}$.

According to the COSMIC database ${ }^{29}$, there are four mutational hotspots in GNAQ: Q209, R183, T96 and Y101. The frequency of these mutation hotspots within GNAQ appears to exhibit significant differences between solid and hematopoietic tumors. Q209 mutations comprise the most frequently mutated hotspot in solid tumors, followed by R183 mutations. In striking contrast, T96 is the predominantly mutated hotspot in hematopoietic tumors, followed by Y101 (Supplementary Fig. 9). This pattern was also verified in NKTCL patients in the present study. This work thus suggests the intriguing possibility that different GNAQ mutations may have different functional consequences, and the properties crucial for their oncogenic functions vary depending on the tissue of origin.

However, this study has some limitations that must be noted. First, the Y101X mutation harbors a premature termination codon. In contrast to T96S, cell line transduction experiments showed that the Y101X mutation could disrupt truncated Gaq protein expression (Supplementary Fig. 10). We speculated that instability of truncated Gaq protein or nonsense-mediated 
mRNA decay might account for the disruption of truncated Gaq protein expression, which needs to be further investigated. We did not conduct functional analysis of the non-hot spot mutants (K57T and M59L) in the present study, given that these two mutants occurred in only $1 \%$ of the NKTCL cases. However, the functions of the two mutants need to be addressed in future studies. Second, germline DNA was unavailable in 26 specimens among the 127 NKTCL cases. Although a strict filter was applied for variant calling, the somatic nature of these mutations identified in this cohort could not be confirmed. We therefore excluded these cases in the subsequent survival analysis. Third, further studies are warranted to clarify the molecular mechanisms mediated by Gaq in suppressing AKT and MAPK signaling in NK cells.

Taken together, we identified recurrent somatic GNAQ T96S mutations, which may contribute to the pathogenesis of NKTCL. These findings have implications for refining our understanding of the genetic mechanisms of NKTCL and for treatment development.

\section{Methods}

Patients and samples. The demographics and clinical features of the patient cohort are summarized in Supplementary Table 1. In total, 127 formalin-fixed, paraffin-embedded (FFPE) tumor tissues from NKTCL patients were obtained at the time of diagnosis at the First Affiliated Hospital of Zhengzhou University. All cases were reviewed and interpreted independently by three experienced pathologists, and diagnoses were made according to the current World Health Organization classification criteria. The experimental design is depicted in Supplementary Fig. 1. The study was conducted in accordance with the Declaration of Helsinki and with approval from the Institutional Review Board of the First Affiliated Hospital of Zhengzhou University. Signed informed consent was obtained from these patients.

DNA extraction. Genomic DNA from whole blood and FFPE samples was extracted using a Blood \& Cell Culture DNA Kit (Qiagen, Hilden, Germany) and a QIAamp DNA FFPE Tissue Kit (Qiagen), respectively. The quality and yield of purified DNA were assessed by fluorimetry (Qubit, Invitrogen), Nanodrop 1000 spectrophotometry (Thermo Scientific, Wilmington, DE, USA), and gel electrophoresis.

Whole-exome sequencing and bioinformatics analysis. To identify somatic genomic variants associated with NKTCL, we performed WES on 28 tumor samples and their matched whole-blood samples. Genomic DNA $(1-1.5 \mu \mathrm{g})$ was fragmented with a Covaris ultrasonicator targeting peak sizes ranging from 180 to $280 \mathrm{bp}$. The fragment ends were blunted and 5'-phosphorylated with T4 polynucleotide kinase, T4 DNA polymerase, and Klenow Large Fragment (New England BioLabs). The 3' ends were A-tailed using Klenow Exo-Minus (New England BioLabs), and the fragments were ligated to Illumina paired-end adapters. Ligation products were purified with Agencourt AMPure XP beads and enriched by PCR using the Illumina PCR primers InPE1.0 and InPE2.0 and PCR primer indices. Pooled, indexed libraries were captured using the Agilent SureSelect Human All Exon $50 \mathrm{Mb}$ kit (Agilent Technologies) according to the manufacturer's protocol and sequenced on an Illumina HiSeq 2500 instrument.

Sequencing reads were aligned to the human reference genome (hg19, downloaded from the UCSC Genome Browser http://genome.ucsc.edu/) using the Burrows-Wheeler Aligner (BWA) ${ }^{56}$ version 0.5.8 with default parameter settings. SAMtools was used to convert the SAM files into BAM files and to pile up sequences after local alignment ${ }^{57}$. The Picard command (http://sourceforge.net/ projects/picard/files/picard-tools/) was used to remove PCR duplications. Next, insertion or deletion (InDel) realignment and base quality score recalibration were performed with the Genome Analysis Toolkit (GATK) version 2.6.5 ${ }^{58}$. For tumor-normal tissue sample pairs, somatic single-nucleotide variations (SNVs) and somatic InDels were called using MuTect ${ }^{59}$ and Strelka ${ }^{60}$, respectively. Control-FREEC was used to detect copy number alterations in tumors compared with normal cells ${ }^{61}$. Somatic copy number alteration (Supplementary Table 14) and loss of heterozygosity (LOH, Supplementary Table 15) analyses were performed in the 28 exome cases. For the 26 non-paired tumor samples with no matched germline DNA, probable somatic variants were detected by SomVarIUS with the default settings ${ }^{62}$. Only variants from the 26 non-paired tumor samples were further filtered to remove those that were present in dbSNP v135 (http://www.ncbi. nlm.nih.gov/SNP/), the 1000 Genomes Project (http://browser.1000genomes.org), or an in-house database containing germline variants identified in $\sim 500$ Chinese exomes. Variants were also visually inspected with the Integrative Genomics Viewer (IGV, https://www.broadinstitute.org/igv/) to exclude probable sequencing artifacts. Gene mutation annotation of the identified variants was carried out using
ANNOVAR ${ }^{63}$. The impact of the SNVs on protein function was predicted by PolyPhen-2, SIFT, or MutationTaster. Whole-exome sequencing data were deposited into the NCBI Sequence Read Archive under accession code SRP107053.

Targeted deep sequencing. We designed a custom panel of 221 genes (Supplementary Table 5) using the SureDesign Tool (Agilent Technologies). Sequencing libraries were prepared from DNA extracted from 73 paired tumor and normal samples using the SureSelect XT2 Target Enrichment System for the Illumina Multiplexed Sequencing Platform (Illumina) according to the manufacturer's instructions. Target-enriched libraries were then sequenced on an Illumina HiSeq 2500 sequencing platform. A bioinformatics analysis was performed as described for the exome sequencing analysis. All candidate variants were manually inspected in the IGV to exclude false positives.

Sanger sequencing. Selected SNVs detected by whole-exome sequencing were validated by Sanger sequencing. Primers specific to the regions of interest were designed and synthesized by Sangon Biotech (Shanghai, China, Supplementary Table 4). PCR was performed using standard procedures followed by direct sequencing on an ABI 3730xl automatic sequencer (PE Applied Biosystems, Foster City, CA, USA).

Mice and genotyping. Ncr1-Cre-Gnaqflfl mice (C57BL/6) were generated by crossing Gnaq ${ }^{f l / f l}$ mice with Ncr1-Cre mice. Gnaq $q^{f l / l}$ mice were generated by Viewsolid Biotech (Beijing, China). Briefly, loxP sites flanking exon 6 of Gnaq were introduced by CRISPR/Cas9. This locus of Gnaq has been previously successfully used to generate conditional Gnaq-deficient mice ${ }^{64}$. The in vitro-synthesized sgRNA, Cas 9 mRNA, and donor vectors were injected into mouse zygotes and then transferred into pseudopregnant mice. Neonatal mutant mice were identified by genotyping and sequencing. Genomic DNA samples were prepared from tail biopsies of 5-day-old mice. PCR genotyping was performed. The primer pairs used to identify correct insertion of LoxP flanking exon 6 of Gnaq and Cre are provided in Supplementary Table 13. The relevant Animal Ethics and Experimentation Committees approved animal experiments according to the guidelines of the First Affiliated Hospital of Zhengzhou University. Both female and male mice aged between 8 and 15 weeks of age were used in this study. Age and sex matching was performed for each independent experiment ( $n \geq 4$ mice per genotype). All procedures were conducted in accordance with the Animal Care and Use Committee guidelines of Zhengzhou University.

To isolate cells from the spleen, spleens were minced and treated with $2 \mathrm{mg} / \mathrm{ml}$ collagenase D in Hanks balanced salt solution with $\mathrm{CaCl}_{2} / \mathrm{MgCl}_{2}$ for $30 \mathrm{~min}$ at room temperature followed by filtering through $70-\mu \mathrm{m}$ nylon cell strainers. The livers of mice were perfused with PBS to remove blood for the isolation of liverresident cells, and single-cell suspensions were subsequently generated as described for the spleen. Single-cell suspensions from lymph nodes were generated by meshing the organs through $70-\mu \mathrm{m}$ nylon cell strainers. Contaminating erythrocytes in cell suspensions were lysed using ACK lysis buffer (Thermo Scientific, Waltham, MA, USA) or removed by density-gradient centrifugation through Ficoll/Hypaque (Sigma-Aldrich, St Louis, MO, USA), and the remaining cells were washed and subjected to subsequent analysis and functional assays. Purified NK cells were obtained by negative selection using NK Cell Isolation kits (Miltenyi Biotec). The purity as assessed by flow cytometry was $90-95 \%$.

For in vivo bromodeoxyuridine (BrdU) incorporation assays, mice were intraperitoneally injected with $2 \mathrm{mg}$ of $\mathrm{BrdU}$ (in $200 \mu \mathrm{l}$ ). After $12 \mathrm{~h}$, splenocytes were isolated, stained, fixed, permeabilized, and treated with DNase. Analysis of BrdU incorporation was performed using a BrdU Flow Kit (BD Pharmingen). Survival was assessed by culturing freshly isolated splenic NK cells for $6 \mathrm{~h}$ at a density of $3 \times 10^{4}$ cells per well in $100 \mu$ of RPMI 1640 medium plus FBS (without IL-2) in 96-well plates. Live and dead cells were discriminated by staining with propidium iodide and Annexin V-FITC (BD Pharmingen). Stained samples were analyzed using FACSCantoII and FACSDiva software Version 6.1.2 (BD Biosciences).

Cell culture. Three NK cell lines ( $\mathrm{YT}^{65}, \mathrm{KHYG1}^{32}$, and NKYS ${ }^{66}$ ) were obtained from Dr. Wing C. Chan (City of Hope Medical Center). The NK92 ${ }^{67}$ cell line was purchased from the American Type Culture Collection (ATCC), and the NKL ${ }^{68}$ cell line was purchased from the cell bank of the Bena Culture Collection (Beijing, China). The SNK6 ${ }^{69}$ cell line was kindly provided by Dr. Norio Shimizu and Yu Zhang of Chiba University. Cells were cultured in RPMI 1640 medium (Invitrogen California, USA) supplemented with 10\% heat-inactivated FBS (Sigma-Aldrich, St Louis, MO, USA), $2 \mathrm{mM}$ glutamine, $100 \mathrm{U} / \mathrm{ml}$ penicillin, and $100 \mu \mathrm{g} / \mathrm{ml}$ streptomycin (Invitrogen, California, USA) in $5 \% \mathrm{CO}_{2}$ atmosphere at $37^{\circ} \mathrm{C}$. In addition, all cell lines except the YT line were interleukin-2 $(150 \mathrm{IU} / \mathrm{ml}$, PeproTech, Rocky Hill, NJ, USA)-dependent. The identity of these cell lines was confirmed by short tandem repeat (STR) profiling or high-throughput sequencing, and they both tested negative for mycoplasma contamination using a Lonza MycoAlert Mycoplasma Detection Kit.

Mutagenesis and constructs. Full-length human GNAQ cDNA was purchased from Sino Biological Inc. (Beijing, China). Mutagenesis to create constructs 
encoding the T96S or Y101X mutants was carried out using a Quick-Change SiteDirected Mutagenesis Kit (Agilent Technologies, Santa Clara, CA) according to the manufacturer's instructions. These constructs encoding wild-type and mutant GNAQ were subcloned into the lentivirus-based expression vector $\mathrm{pCDH}-\mathrm{CMV}$ MCS-EF1-copGFP (System Biosciences, \#CD511B-1). In addition, $3 \times$ Flag-tagged GNAQ T96S was subcloned into the tetracycline-inducible pLVX-TetOne-Puro vector (Clontech, Takara, \#631849). All cDNA sequences were confirmed by Sanger sequencing.

Lentivirus production and generation of stable cell lines. For overexpression of wild-type GNAQ and mutants, $12 \mu \mathrm{g}$ of purified plasmids pCDH-CMV-MCS-EF1copGFP-vector, pCDH-CMV-MCS-EF1-copGFP-wild-type, pCDH-CMV-MCSEF1-copGFP-T96S, or pCDH-CMV-MCS-EF1-copGFP-Y101X was cotransfected with $8 \mu \mathrm{g}$ of psPAX2 packaging plasmid and $4 \mu \mathrm{g}$ of pMD2.G envelope plasmid into HEK293T cells using Lipofectamine 2000 (Invitrogen) according to the manufacturer's protocol. The viruses were harvested $72 \mathrm{~h}$ posttransfection, and cells were infected with these viruses in the presence of $8 \mathrm{mg} / \mathrm{ml}$ polybrene. Infected cells expressing GFP were sorted using a flow cytometer (FACSCantoII, BD Bioscience, San Jose, CA, USA), and the purity of the sorted cell fractions consistently exceeded $95 \%$.

To establish cell lines stably expressing equal amounts of wild-type and T96S simultaneously, KHYG1 and YT cells were infected with PCDH-CMV-MCS-EF1copGFP-vector or pCDH-CMV-MCS-EF1-copGFP-wild-type lentivirus. The stably transfected clones were selected by GFP. These cells were subsequently infected with pLVX-TetOne-Puro-vector or pLVX-TetOne-Puro-3×Flag-T96S lentivirus and selected with $4 \mu \mathrm{g} / \mathrm{ml}$ puromycin (Sigma Chemical Co., St Louis, MO, USA) to establish tetracycline-inducible cell lines. The tetracycline-inducible cell lines were maintained in RPMI 1640 medium with $0.25 \mu \mathrm{g} / \mathrm{ml}$ puromycin, and the cells were incubated with $20 \mathrm{ng} / \mathrm{ml}$ doxycycline (Sigma-Aldrich, Shanghai, China) for up to $48 \mathrm{~h}$ to induce T96S mutant expression.

Cell viability assay. Cells were seeded in $96-$ well plates in triplicate at a density of $5 \times 10^{3}$ cells/well in $100 \mu$ l of cell culture medium. Cell viability was evaluated using a Cell Counting Kit-8 (CCK-8, Dojindo, Tokyo, Japan) at the indicated days according to the manufacturer's instructions. In brief, $10 \mu \mathrm{l}$ of CCK-8 reagent was added to each well. The cells were incubated at $37^{\circ} \mathrm{C}$ in an atmosphere containing $5 \% \mathrm{CO}_{2}$ for $2 \mathrm{~h}$. Cell viability was presented by the absorbance value at OD $450 \mathrm{~nm}$, which was measured with a Multiskan FC microplate reader (Thermo Scientific, Waltham, MA, USA). The value is directly proportional to the number of viable cells in the culture medium. Independent experiments were repeated at least three times.

Flow cytometric analysis. Cell proliferation was assessed by BrdU incorporation assays with a BrdU Flow Kit (BD Pharmingen) or an EdU Click Proliferation Kit (BD Pharmingen). Cell apoptosis was analyzed with a PE-Annexin V Kit (BD Pharmingen). The following antibodies were purchased from BD Biosciences: CD122-PE (TM-Bta1), CD19-FITC (1D3), CD335/NKp46-PE (29A1.4), CD3eFITC (145-2C11), CD3e-PerCP-Cy5.5 (145-2C11), CD4-FITC (RM4-5), CD49b (DX5) Pan-NK Cells-BV421, CD8a-PE (53-6.7), Erythroid Cells-FITC (TER-119), Ly-6G/Ly-6C-FITC (RB6-8C5), NK-1.1-APC (PK136), NK-1.1-APC-Cy7 (PK136), and TCR-Bta Chain-BV421 (H57-597). Stained samples were analyzed using FACSCantoII and FACSDiva software Version 6.1.2 (BD Biosciences).

Xenograft tumor assay. Female NOD/SCID (4-5 weeks old, No. 11400700201639) mice were purchased from Vital River Laboratories (Beijing, China). The mice were housed five/cage in microisolator units under humidityand temperature-controlled conditions with 12 -h light-dark cycles. The animals were fed a standard sterile laboratory diet (Zhengzhou University, Zhengzhou, China) beginning at least 3 days before use. Animal health was examined prior to tumor implantation and randomization to ensure that only animals without any symptoms of disease were selected to begin experimental procedures. During the experiments, animals were monitored twice daily regarding tumor burden, general conditions, and food and water supply. The mice were randomly distributed into four groups and subcutaneously injected with $5.0 \times 10^{6}$ cells in $0.1 \mathrm{ml}$ of PBS/ Matrigel (BD Biosciences, CA, USA) mixture (1:1 volume) into the flank regions. These four groups were tetracycline-inducible YT cells stably transfected with vector control, wild-type, T96S, and wild-type + T96S ( $n=5$ per group). When the needle was inserted under the skin, it was held parallel to the animal's body to avoid puncturing underlying structures, and the syringe was aspirated to ensure that the needle tip had not penetrated a blood vessel. Doxycycline was administered as a $2 \mathrm{mg} / \mathrm{ml}$ concentration in drinking water supplemented with $5 \%$ sucrose (Sigma-Aldrich, Shanghai, China). Because doxycycline has limited stability in water, the supply was changed every other day. The tumor size was measured every 2 days with calipers, and the tumor burden was monitored after 6 weeks of implantation by utilizing the IVIS Spectrum system (Perkin Elmer, Beaconsfield, UK). The mice were then euthanized by asphyxiation in a $\mathrm{CO}_{2}$ chamber, and the tumors were excised using standard forceps, scissors, and surgical blades. For histological analysis of xenograft tumors, mouse samples were blinded during sample processing and just identified by their mouse ID numbers. All procedures were conducted in accordance with the Animal Care and Use Committee guidelines of Zhengzhou University.

TUNEL assay. Xenograft tumors were fixed in 10\% PBS-buffered formalin and embedded in paraffin. The apoptotic cells were determined by TUNEL staining using a One Step TUNEL Apoptosis Assay Kit (Beyotime, Jiangsu, China), and the nuclei were stained with DAPI. Images were captured using a fluorescence microscope (DMI400B, Leica Company, Germany).

RNA sequencing and analysis. RNA isolation, library construction, and sequencing were performed by the Novogene Bioinformatics Institute (Beijing, China). Total RNAs were extracted using the RNeasy Mini kit (Qiagen) according to the manufacturer's protocol. The quality and yield of the RNA were assessed by fluorimetry (Qubit, Invitrogen), Nanodrop 1000 spectrophotometry (Thermo Scientific, Wilmington, DE, USA), and gel electrophoresis. RNA integrity was assessed using the RNA Nano 6000 Assay kit with the Bioanalyzer 2100 system (Agilent Technologies, CA, USA). A total of $3 \mu \mathrm{g}$ of RNA from each sample was used as input material for RNA sample preparation. First, ribosomal RNA was removed using an rRNA Removal kit (Epicentre, WI, USA). Second, sequencing libraries were generated using the Illumina TruSeq RNA Sample Preparation kit (Illumina, CA, USA) according to the manufacturer's recommendations. To select cDNA fragments between 150 and $200 \mathrm{bp}$ in length, the library fragments were purified with the AMPure XP system (Beckman Coulter, Beverly, USA). Fragments were then amplified by ten cycles of PCR using Phusion DNA polymerase, and libraries were validated with the Bioanalyzer 2100 system (Agilent Technologies, CA, USA). Lastly, the libraries were applied to an Illumina flow cell using the Illumina Cluster Station. After cluster generation, the libraries were sequenced on an Illumina Hiseq 2000 platform, and 100-bp paired-end reads were generated. Bowtie (v2.2.3) was used to build the reference genome index, and TopHat (v2.0.12) was used to align the clean paired-end reads to the reference genome. The read numbers mapped to each gene were counted by HTSeq (v0.6.1), and the expected number of fragments per kilobase of transcript sequence per million base pairs sequenced (FPKM) was calculated. GSEA (version 2.2.4) and gene set collection of KEGG pathways were used for enrichment analysis. The statistical significance of signature enrichment was assessed using 1000-gene-set permutations The raw sequencing data are available from the NCBI and are archived under the accession number SRP180943.

Immunoprecipitation. The immunoprecipitation assay was performed using the Pierce $^{\mathrm{mt}}$ Classic Magnetic IP/Co-IP kit (Thermo Scientific, Waltham, MA, USA) according to the manufacturer's protocol. Briefly, cells $\left(1.0 \times 10^{7}\right)$ were lysed in cold Pierce IP Lysis buffer and Halt ${ }^{\mathrm{m}}$ Protease and Phosphatase Inhibitor cocktail (Thermo Scientific, Waltham, MA, USA) for $15 \mathrm{~min}$ on ice. Cell debris was removed by centrifugation at $13,000 \times \mathrm{g}$ for $10 \mathrm{~min}$. Cell lysate $(1000 \mu \mathrm{g})$ was combined with $10 \mu \mathrm{g}$ of Flag antibody (Thermo Scientific, Waltham, MA, USA) and incubated overnight at $4{ }^{\circ} \mathrm{C}$ with rotation. The antigen sample/antibody mixture was added to the tube containing prewashed magnetic beads $(50 \mu \mathrm{l})$ and incubated at room temperature for $1 \mathrm{~h}$ with mixing. The beads were collected with a magnetic stand and washed twice with IP lysis/wash buffer and once with purified water; the antigen/antibody complex was then eluted.

Mass spectrometry analysis. The eluted proteins were digested according to the SISPROT protocol, as described in a previous study ${ }^{70}$. The desalted peptides were lyophilized under vacuum and kept at $-20^{\circ} \mathrm{C}$ until mass spectrometry analysis.

The desalted sample was dissolved in solvent A and analyzed via reverse-phase high-pressure liquid chromatography electrospray ionization tandem mass spectrometry (RP-HPLC-ESI-MS/MS) using a TripleTOF 5600+ mass spectrometer (SCIEX, Concord, Ontario, Canada) coupled to a NanoSpray III ion source and a nanoLC Eksigent 415 system (SCIEX, Concord, Ontario, Canada). Nano-scale reverse-phase liquid chromatography (RPLC) was performed with a trap-and-elution configuration using a Nano cHiPLC Trap column $(200 \mu \mathrm{m} \times 0.5$ mm ChromXP C18-CL $3 \mu \mathrm{m} 120 \AA$ ) and a nano-scale analytical column $(75 \mu \mathrm{m} \times$ $15 \mathrm{~cm}$ ChromXP C18-CL $3 \mu \mathrm{m} 120 \AA$ ). Solvents A and B were $2 \%$ and $98 \%$ acetonitrile in water supplemented with $0.1 \%$ formic acid. The sample was loaded in the trap column at a flow rate of $3 \mu \mathrm{l} / \mathrm{min}$ for $7 \mathrm{~min}$ using $100 \%$ solvent A. For each sample, a stepwise gradient of $60 \mathrm{~min}(0-0.5 \mathrm{~min}, 95-92 \% \mathrm{~A} ; 0.5-38 \mathrm{~min}$, 92-75\% A; 38-44 min, 75-65\% A; 44-45 min, 65-20\% A; $45-51$ min, $20 \%$ A $51-52 \mathrm{~min}, 20-95 \% \mathrm{~A} ; 52-60 \mathrm{~min}, 95 \% \mathrm{~A})$ or $90 \mathrm{~min}(0-0.5 \mathrm{~min}, 95-93 \% \mathrm{~A}$; 0.5-55 min, $93-75 \%$ A; 55-70 min, $75-65 \%$ A; 70-75 min, 65-50\% A; 75-76 min, $50-20 \%$ A; $76-81 \mathrm{~min}, 20 \%$ A; $81-82 \min , 20-95 \%$ A; $82-90 \min , 95 \%$ A) was separately performed using a flow rate of $300 \mu \mathrm{l} / \mathrm{min}$.

The following parameters were used with the mass spectrometer: MS survey scan range, $350-1500 \mathrm{~m} / z$; MS survey, $0.25 \mathrm{~s}$; MS/MS scan range, $100-1500 \mathrm{~m} / \mathrm{z}$. The precursor ions were fragmented in the collision cell using rolling collision energy, and the collision energy spread (CES) was set to 10 . Up to 30 precursor ions were selected for subsequent MS/MS experiments with an accumulation time of $0.07 \mathrm{~s}$ per MS/MS scan and a total cycle time of $2.4 \mathrm{~s}$. The selection criteria for precursor ions included an intensity greater than $200 \mathrm{cps}$ and a charge state ranging from +2 to +5 . The precursor ions were fragmented in the collision cell using 
rolling collision energy, and the CES was set to 5 . The following parameters were used: dynamic excluded time, $18 \mathrm{~s}$; ignore peaks within $6 \mathrm{Da}$; exclude after occurrences, 1; mass tolerance, $50 \mathrm{ppm}$.

The original .wiff files were converted to .mgf files using PeakView software (SCIEX, Concord, Ontario, Canada), and the resulting files were subjected to database searches using Mascot 2.3 (Matrix Science, London, United Kingdom). The following parameters were used for database search: trypsin, KR/P; max cleavage sites, 2; fixed modification, carboxamidomethylation (C); variable modification, oxidation $(\mathrm{M})$, deamidated $(\mathrm{NQ})$, acetyl (N-term); mass tolerance, $0.05 \mathrm{Da}$ for the precursor ion and $0.1 \mathrm{Da}$ for the fragment ion. The searches were conducted in a UniProt Swiss-Prot database (downloaded in May 2017, with 20201 reviewed and canonical protein sequence entries) containing whole Homo sapiens proteins and the same number of reversed protein sequences. Three independent IP-Mass experiments were performed. SAINT express (v3.6.1) was the statistical tool utilized to calculate the probability of protein-protein interaction from background, nonspecific interactions with a SAINT probability threshold of $\geq 0.8$ (ref. ${ }^{34-37}$ ).

Western blot analysis. Cells were lysed in cold RIPA lysis buffer and Halt ${ }^{\text {tw }}$ Protease and Phosphatase Inhibitor cocktail (Thermo Scientific, Waltham, MA, USA) for $20 \mathrm{~min}$ on ice. The cell lysates were clarified by centrifugation at $10,000 \times g$ for $20 \mathrm{~min}$. Proteins $(10-25 \mu \mathrm{g})$ were resolved by SDS-PAGE and transferred onto nitrocellulose membranes (Amersham Biosciences, Piscataway, NJ, USA). The membranes were blocked in TBS-T buffer $(20 \mathrm{mM}$ Tris-HCl, $\mathrm{pH}$ 7.5, $150 \mathrm{mM}$ $\mathrm{NaCl}$, and $0.05 \%$ Tween-20) containing $5 \%(\mathrm{w} / \mathrm{v})$ non-fat milk at room temperature for $1 \mathrm{~h}$ and then probed at $4{ }^{\circ} \mathrm{C}$ overnight with antibodies to detect AKT (pan) (clone C67E7, 1:1000), phospho-AKT (Ser473) (clone D9E, 1:1000), phospho-p44/ 42 MAPK (ERK1/2) (Thr202/Tyr204) (clone D13.14.4E, 1:1000), p44/42 MAPK (ERK1/2) (clone 137F5, 1:2000) from Cell Signaling Technology (Boston, MA, USA); Gaq (clone ab75825 and ab190082, 1:1000) from Abcam (Cambridge, USA); DYKDDDDK Tag Monoclonal Antibody (FG4R, MA1-91878, 1:1000) from Thermo Scientific (Waltham, MA, USA), and GAPDH (clone 60004-1-Ig, 1:2000) from ProteinTech (Chicago, IL, USA). Detection was carried out with the SuperSignal West Femto Maximum Sensitivity Substrate Trial kit (Pierce, Rockford, IL, USA). The band images were digitally captured and quantified with a ChemiDoc ${ }^{\mathrm{m}}$ XRS+ system (Bio-Rad Laboratories, Hercules, CA, USA).

Immunohistochemistry. Immunohistochemistry was performed as described previously $^{71}$. The FFPE sections were immunostained using the Dako EnVision ${ }^{\text {ma }}$ Flex+ System (K8012; Dako, Glostrup, Denmark). Deparaffinization and epitope unmasking were carried out in a PT Link instrument using an EnVision ${ }^{\text {to }}$ Flex target retrieval solution (Dako, Carpinteria, CA, USA). The sections were treated with $0.3 \%$ hydrogen peroxide $\left(\mathrm{H}_{2} \mathrm{O}_{2}\right)$ for $5 \mathrm{~min}$ to block endogenous peroxidase. Sections were incubated overnight at $4{ }^{\circ} \mathrm{C}$ with the following antibodies: phosphop44/42 MAPK (ERK1/2) (Thr202/Tyr204) (clone D13.14.4E; diluted 1:100; Cell Signaling Technology, Beverly, MA), phospho-AKT (Ser473) (clone D9E; diluted 1:100; Cell Signaling Technology, Beverly, MA), Gaq (clone ab75825; diluted 1:50; Abcam), and Ki67 (clone MIB-1; diluted 1:50; Dako). The specimens were subsequently treated with EnVision ${ }^{\text {tw }}$ Flex mouse or rabbit linker (15 min), EnVision ${ }^{\text {now }}$ Flex/HRP enzyme (30 min), and 3'3-diaminobenzidine tetrahydrochloride (10 min). The samples were counterstained with hematoxylin, dehydrated, and mounted with Richard-Allan Scientific Cyto seal XYL mounting medium (Thermo Scientific, Waltham, MA, USA). The sample series included appropriate positive and negative controls. All cases were scored by the pathologist without prior knowledge of the patients' mutational status and outcomes. For histological analysis of xenograft tumors, mouse samples were blinded during sample processing and just identified by their mouse ID numbers. Expression levels of p-ERK and pAKT were scored using the semi-quantitative $H$-score (Histochemical score) visual approach, taking into consideration the intensity of staining and the percentage of stained cells $\mathrm{s}^{72}$. Staining intensity was scored as $0,1,2$, or 3 for negative, weak, moderate, and strong, respectively. The percentage of positive cells for each intensity was subjectively estimated. $H$-score ranged from 0 (no staining) to 300 (maximum staining) and was calculated by application of the following formula: $H$ score $=1 \times(\%$ light staining $)+2 \times(\%$ moderate staining $)+3 \times(\%$ strong staining). Alternatively, expression levels of p-ERK and p-AKT were scored semiquantitatively according to the percentage of IHC-positive cell:,$+ 0 ;++, 1-10 \%$; ,$+++ 11-50 \%$;,$++++>50 \%$. All cases were scored by the pathologist without prior knowledge of the patients' mutational status of Gaq. The relationship between the expression of p-ERK (or p-AKT) and Gaq mutational status were evaluated by Fisher's exact test, $2 \times 4$ table (www.vassarstats.net).

Statistical analysis. All data are expressed as the mean \pm s.e.m. Comparisons between and among groups were performed with unpaired two-tailed Student's $t$-test and analysis of variance (ANOVA), respectively. The association of GNAQ mutations and clinicopathological characteristics was analyzed by the $\chi^{2}$ test or Fisher's two-tailed exact test. Analyses were conducted to evaluate the relationship between gene mutations and prognosis, and only those frequently mutated genes (mutated in at least 5\% of patients) were included. OS rates and PFS were calculated using the Kaplan-Meier method, and significance was assessed by the log-rank test. Derived $P$ values were adjusted for false discovery rate (FDR) using the Benjamini-Hochberg method ${ }^{73}$, and an FDR threshold of 0.1 was set for significance. Statistical analysis was carried out using IBM SPSS Statistics 19 software (IBM Corp., Armonk, NY, USA) and the online statistics calculator VassarStats (www.vassarstats.net). $P<0.05$ was considered significant.

\section{Data availability}

The data supporting the main findings of this study are available within the article and its Supplementary Information and Supplementary Data files. Whole-exome sequencing data were deposited into the NCBI Sequence Read Archive under accession code SRP107053 (https://www.ncbi.nlm.nih.gov/sra/). The raw RNA sequencing data are available from NCBI and are archived under accession number SRP180943 (https://www. ncbi.nlm.nih.gov/sra/). The raw LC/MS data have been deposited to the ProteomeXchange Consortium ${ }^{74}$ via the PRIDE $^{75}$ partner repository with the dataset identifier PXD013285 (www.ebi.ac.uk/pride). The source data underlying Figs. 3b-f, $4 b-f, 5 a-d, f$, and $6 b, c$, e and Supplementary Fig. 6 are provided as a Source Data file. Any other data are available from the authors upon reasonable request.

Received: 16 November 2017 Accepted: 16 August 2019

Published online: 16 September 2019

\section{References}

1. Aviles, A., Diaz, N. R., Neri, N., Cleto, S. \& Talavera, A. Angiocentric nasal T/ natural killer cell lymphoma: a single centre study of prognostic factors in 108 patients. Clin. Lab. Haematol. 22, 215-220 (2000).

2. Barrionuevo, C. et al. Extranodal NK/T-cell lymphoma, nasal type: study of clinicopathologic and prognosis factors in a series of 78 cases from Peru. Appl. Immunohistochem. Mol. Morphol. 15, 38-44 (2007).

3. Kwong, Y. L. Natural killer-cell malignancies: diagnosis and treatment. Leukemia 19, 2186-2194 (2005)

4. Gualco, G. et al. Clinicopathologic and molecular features of 122 Brazilian cases of nodal and extranodal NK/T-cell lymphoma, nasal type, with EBV subtyping analysis. Am. J. Surg. Pathol. 35, 1195-1203 (2011).

5. Li, S. et al. Extranodal NK/T-cell lymphoma, nasal type: a report of 73 cases at MD Anderson Cancer Center. Am. J. Surg. Pathol. 37, 14-23 (2013).

6. Peng, R. J. et al. Genomic and transcriptomic landscapes of Epstein-Barr virus in extranodal natural killer T-cell lymphoma. Leukemia 33, 1451-1462 (2019).

7. Kawa, K. [Clinical characteristics of Epstein-Barr virus-associated natural killer cell lymphoma/leukemia]. Nihon Rinsho Jpn. J. Clin. Med. 55, 424-428 (1997).

8. Nava, V. E. \& Jaffe, E. S. The pathology of NK-cell lymphomas and leukemias. Adv. Anat. Pathol. 12, 27-34 (2005).

9. Laurini, J. A. et al. Classification of non-Hodgkin lymphoma in Central and South America: a review of 1028 cases. Blood 120, 4795-4801 (2012).

10. Jaccard, A. et al. Efficacy of L-asparaginase with methotrexate and dexamethasone (AspaMetDex regimen) in patients with refractory or relapsing extranodal NK/T-cell lymphoma, a phase 2 study. Blood 117, 1834-1839 (2011).

11. Tse, E. \& Kwong, Y. L. How I treat NK/T-cell lymphomas. Blood 121, 4997-5005 (2013).

12. Lim, S. T. et al. Comparative analysis of extra-nodal NK/T-cell lymphoma and peripheral T-cell lymphoma: significant differences in clinical characteristics and prognosis. Eur. J. Haematol. 80, 55-60 (2008).

13. Chan, J. Y. \& Lim, S. T. Novel findings from the Asian Lymphoma Study Group: focus on $\mathrm{T}$ and NK-cell lymphomas. Int. J. Hematol. 107, 413-419 (2018).

14. de Mel, S. et al. The genomics and molecular biology of natural killer/T-cell lymphoma: opportunities for translation. Int. J. Mol. Sci. 19, 1931 (2018).

15. Kucuk, C. et al. Activating mutations of STAT5B and STAT3 in lymphomas derived from gammadelta-T or NK cells. Nat. Commun. 6, 6025 (2015).

16. Huang, Y. et al. Gene expression profiling identifies emerging oncogenic pathways operating in extranodal NK/T-cell lymphoma, nasal type. Blood 115, 1226-1237 (2010)

17. Ng, S. B. et al. Dysregulated microRNAs affect pathways and targets of biologic relevance in nasal-type natural killer/T-cell lymphoma. Blood 118, 4919-4929 (2011).

18. Ng, S. B. et al. Activated oncogenic pathways and therapeutic targets in extranodal nasal-type NK/T cell lymphoma revealed by gene expression profiling. J. Pathol. 223, 496-510 (2011).

19. Iqbal, J. et al. Natural killer cell lymphoma shares strikingly similar molecular features with a group of non-hepatosplenic gammadelta T-cell lymphoma and is highly sensitive to a novel aurora kinase A inhibitor in vitro. Leukemia $\mathbf{2 5}$ 348-358 (2011). 
20. Song, T. L. et al. Oncogenic activation of the STAT3 pathway drives PD-L1 expression in natural killer/T-cell lymphoma. Blood 132, 1146-1158 (2018).

21. Chan, J. Y. et al. Whole exome sequencing identifies recessive germline mutations in FAM160A1 in familial NK/T cell lymphoma. Blood Cancer J. 8, 111 (2018).

22. Dufva, O. et al. Aggressive natural killer-cell leukemia mutational landscape and drug profiling highlight JAK-STAT signaling as therapeutic target. Nat. Commun. 9, 1567 (2018).

23. Wen, H. et al. Recurrent ECSIT mutation encoding V140A triggers hyperinflammation and promotes hemophagocytic syndrome in extranodal NK/T cell lymphoma. Nat. Med. 24, 154-164 (2018)

24. Lee, S. et al. Genetic alterations of JAK/STAT cascade and histone modification in extranodal NK/T-cell lymphoma nasal type. Oncotarget 6, 17764-17776 (2015).

25. Koo, G. C. et al. Janus kinase 3-activating mutations identified in natural killer/T-cell lymphoma. Cancer Discov. 2, 591-597 (2012).

26. Dobashi, A. et al. Frequent BCOR aberrations in extranodal NK/T-Cell lymphoma, nasal type. Genes, Chromosomes Cancer 55, 460-471 (2016).

27. Zhang, Y., Li, C., Xue, W., Zhang, M. \& Li, Z. Frequent mutations in natural killer/T cell lymphoma. Cell. Physiol. Biochem. 49, 1-16 (2018).

28. Li, T. et al. Mutations of the p53 gene in nasal NK/T-cell lymphoma. Lab. Investig. 80, 493-499 (2000).

29. Forbes, S. A. et al. The catalogue of somatic mutations in cancer (COSMIC). Curr. Protoc. Hum. Genet. 57, 10.11.1-10.11.26 (2008).

30. Kern, W. F. et al. Neural cell adhesion molecule-positive peripheral T-cell lymphoma: a rare variant with a propensity for unusual sites of involvement. Blood 79, 2432-2437 (1992).

31. Wong, K. F. et al. CD56 (NKH1)-positive hematolymphoid malignancies: an aggressive neoplasm featuring frequent cutaneous/mucosal involvement, cytoplasmic azurophilic granules, and angiocentricity. Hum. Pathol. 23 798-804 (1992).

32. Yagita, M. et al. A novel natural killer cell line (KHYG-1) from a patient with aggressive natural killer cell leukemia carrying a p53 point mutation. Leukemia 14, 922-930 (2000).

33. MacLeod, R. A. et al. Human leukemia and lymphoma cell lines as models and resources. Curr. Med. Chem. 15, 339-359 (2008).

34. Teo, G. et al. SAINTq: scoring protein-protein interactions in affinity purification - mass spectrometry experiments with fragment or peptide intensity data. Proteomics 16, 2238-2245 (2016).

35. Teo, G. et al. SAINTexpress: improvements and additional features in Significance Analysis of INTeractome software. J. Proteomics 100, 37-43 (2014).

36. Skarra, D. V. et al. Label-free quantitative proteomics and SAINT analysis enable interactome mapping for the human Ser/Thr protein phosphatase 5 Proteomics 11, 1508-1516 (2011).

37. Choi, $H$. et al. SAINT: probabilistic scoring of affinity purification-mass spectrometry data. Nat. Methods 8, 70-73 (2011).

38. Barren, B. \& Artemyev, N. O. Mechanisms of dominant negative G-protein alpha subunits. J. Neurosci. Res. 85, 3505-3514 (2007).

39. Misra, R. S. et al. G alpha q-containing $G$ proteins regulate B cell selection and survival and are required to prevent B cell-dependent autoimmunity. J. Exp. Med. 207, 1775-1789 (2010).

40. Wang, D. et al. The deficiency of Galphaq leads to enhanced T-cell survival. Immunol. Cell Biol. 92, 781-790 (2014).

41. Jiang, L. et al. Exome sequencing identifies somatic mutations of DDX3X in natural killer/T-cell lymphoma. Nat. Genet. 47, 1061-1066 (2015).

42. Kimura, H. \& Fujiwara, S. Overview of EBV-associated T/NK-cell lymphoproliferative diseases. Front. Pediatr. 6, 417 (2018).

43. Kim, S. J., Yoon, S. E. \& Kim, W. S. Treatment of localized extranodal NK/T cell lymphoma, nasal type: a systematic review. J. Hematol. Oncol. 11, 140 (2018).

44. Yamaguchi, M., Oguchi, M. \& Suzuki, R. Extranodal NK/T-cell lymphoma: updates in biology and management strategies. Best Pract. Res. Clin. Haematol. 31, 315-321 (2018).

45. Yamaguchi, M., Suzuki, R. \& Oguchi, M. Advances in the treatment of extranodal NK/T-cell lymphoma, nasal type. Blood 131, 2528-2540 (2018).

46. Kommalapati, A., Tella, S. H., Ganti, A. K. \& Armitage, J. O. Natural killer/Tcell neoplasms: analysis of incidence, patient characteristics, and survival outcomes in the United States. Clin. Lymphoma, Myeloma Leuk. 18, 475-479 (2018).

47. Ng, S. Y. et al. Targetable vulnerabilities in T- and NK-cell lymphomas identified through preclinical models. Nat. Commun. 9, 2024 (2018).

48. Litosch, I. Decoding Galphaq signaling. Life Sci. 152, 99-106 (2016).

49. O'Hayre, M. et al. The emerging mutational landscape of $\mathrm{G}$ proteins and G-protein-coupled receptors in cancer. Nat. Rev. Cancer 13, 412-424 (2013).
50. $\mathrm{Wu}, \mathrm{M}$. et al. Galpha13 negatively controls osteoclastogenesis through inhibition of the Akt-GSK3beta-NFATcl signalling pathway. Nat. Commun. 8, 13700 (2017).

51. Zhang, Z. et al. GNA13 promotes tumor growth and angiogenesis by upregulating CXC chemokines via the NF-kappaB signaling pathway in colorectal cancer cells. Cancer Med. 7, 5611-5620 (2018).

52. Rasheed, S. A. K. et al. GNA13 expression promotes drug resistance and tumor-initiating phenotypes in squamous cell cancers. Oncogene 37, 1340-1353 (2018)

53. Muppidi, J. R. et al. Loss of signalling via Galpha13 in germinal centre B-cellderived lymphoma. Nature 516, 254-258 (2014).

54. O'Hayre, M. et al. Inactivating mutations in GNA13 and RHOA in Burkitt's lymphoma and diffuse large B-cell lymphoma: a tumor suppressor function for the Galpha13/RhoA axis in B cells. Oncogene 35, 3771-3780 (2016).

55. Healy, J. A. et al. GNA13 loss in germinal center B cells leads to impaired apoptosis and promotes lymphoma in vivo. Blood 127, 2723-2731 (2016).

56. Li, H. \& Durbin, R. Fast and accurate short read alignment with BurrowsWheeler transform. Bioinformatics 25, 1754-1760 (2009).

57. Li, H. et al. The Sequence Alignment/Map format and SAMtools. Bioinformatics 25, 2078-2079 (2009).

58. McKenna, A. et al. The Genome Analysis Toolkit: a MapReduce framework for analyzing next-generation DNA sequencing data. Genome Res. 20, 1297-1303 (2010).

59. Cibulskis, K. et al. Sensitive detection of somatic point mutations in impure and heterogeneous cancer samples. Nat. Biotechnol. 31, 213-219 (2013).

60. Saunders, C. T. et al. Strelka: accurate somatic small-variant calling from sequenced tumor-normal sample pairs. Bioinformatics 28, 1811-1817 (2012).

61. Boeva, V. et al. Control-FREEC: a tool for assessing copy number and allelic content using next-generation sequencing data. Bioinformatics 28, 423-425 (2012).

62. Smith, K. S. et al. SomVarIUS: somatic variant identification from unpaired tissue samples. Bioinformatics 32, 808-813 (2016).

63. Wang, K., Li, M. \& Hakonarson, H. ANNOVAR: functional annotation of genetic variants from high-throughput sequencing data. Nucleic Acids Res. 38, e164 (2010).

64. Wettschureck, N. et al. Absence of pressure overload induced myocardial hypertrophy after conditional inactivation of Galphaq/Galpha11 in cardiomyocytes. Nat. Med. 7, 1236-1240 (2001).

65. Drexler, H. G. \& Matsuo, Y. Malignant hematopoietic cell lines: in vitro models for the study of natural killer cell leukemia-lymphoma. Leukemia 14, 777-782 (2000)

66. Tsuchiyama, J. et al. Characterization of a novel human natural killer-cell line (NK-YS) established from natural killer cell lymphoma/leukemia associated with Epstein-Barr virus infection. Blood 92, 1374-1383 (1998).

67. Gong, J. H., Maki, G. \& Klingemann, H. G. Characterization of a human cel line (NK-92) with phenotypical and functional characteristics of activated natural killer cells. Leukemia 8, 652-658 (1994).

68. Robertson, M. J. et al. Characterization of a cell line, NKL, derived from an aggressive human natural killer cell leukemia. Exp. Hematol. 24, 406-415 (1996)

69. Nagata, H. et al. Characterization of novel natural killer (NK)-cell and gammadelta T-cell lines established from primary lesions of nasal T/NKcell lymphomas associated with the Epstein-Barr virus. Blood 97, 708-713 (2001).

70. Bian, Y. et al. Ultra-deep tyrosine phosphoproteomics enabled by a phosphotyrosine superbinder. Nat. Chem. Biol. 12, 959-966 (2016).

71. Li, Z. et al. Recurrent mutations in epigenetic modifiers and the PI3K/AKT/ mTOR pathway in subcutaneous panniculitis-like T-cell lymphoma. $\mathrm{Br}$. J. Haematol. 181, 406-410 (2018).

72. McCarty, K. S., Jr. Miller, L. S., Cox, E. B., Konrath, J. \& McCarty, K. S. Sr. Estrogen receptor analyses. Correlation of biochemical and immunohistochemical methods using monoclonal antireceptor antibodies. Arch. Pathol. Lab. Med. 109, 716-721 (1985).

73. Benjamini, Y. H. Y. Controlling the false discovery rate: a practical and powerful approach to multiple testing. J. R. Stat. Soc. B 57, 289-300 (1995).

74. Deutsch, E. W. et al. The ProteomeXchange consortium in 2017: supporting the cultural change in proteomics public data deposition. Nucleic Acids Res. 45, D1100-D1106 (2017).

75. Perez-Riverol, Y. et al. The PRIDE database and related tools and resources in 2019: improving support for quantification data. Nucleic Acids Res. 47, D442-D450 (2019).

\section{Acknowledgements}

This study was supported by funds from the National Natural Science Foundation of China $(81570203,81172118)$ and Innovation Funds Project of the First Affiliated 
Hospital of Zhengzhou University (to M.Z.). The authors gratefully acknowledge Dr. Tian Tian for critical review and language editing of the manuscript.

\section{Author contributions}

Z.L., W.L. and M.Z. designed and performed the research, analyzed the data, and wrote the paper; Z.L., X.Z., W.X. and Yanjie Zhang executed the experiments and interpreted the data; C.L., Y.S., M.M., Lisha Lu, Y.W., Z.Z., M.J., Y.B., L.Z., X.W., Ling Li, X.L., Xiaorui Fu, Z.S., J.W., F.N., Y.C., Xiaoyan Feng, J.Y. and H.Y. executed the experiments; W.L., G.W., D.Z. and K.H.Y. contributed to the IHC analysis; Xuefei Fu contributed to the sequencing and bioinformatics analysis; Z.L., W.L., M.Z. and Yuan Zhang contributed to the experimental design and analysis.

\section{Additional information}

Supplementary Information accompanies this paper at https://doi.org/10.1038/s41467019-12032-9.

Competing interests: The authors declare no competing interests.

Reprints and permission information is available online at http://npg.nature.com/ reprintsandpermissions/
Peer review information Nature Communications thanks Philippe Gaulard, Ryan Morin and other, anonymous, reviewer(s) for their contribution to the peer review of this work.

Publisher's note Springer Nature remains neutral with regard to jurisdictional claims in published maps and institutional affiliations.

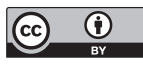

Open Access This article is licensed under a Creative Commons Attribution 4.0 International License, which permits use, sharing, adaptation, distribution and reproduction in any medium or format, as long as you give appropriate credit to the original author(s) and the source, provide a link to the Creative Commons license, and indicate if changes were made. The images or other third party material in this article are included in the article's Creative Commons license, unless indicated otherwise in a credit line to the material. If material is not included in the article's Creative Commons license and your intended use is not permitted by statutory regulation or exceeds the permitted use, you will need to obtain permission directly from the copyright holder. To view a copy of this license, visit http://creativecommons.org/ licenses/by/4.0/.

(C) The Author(s) 2019 